\title{
AIP1-mediated actin disassembly is required for postnatal germ cell migration and spermatogonial stem cell niche establishment
}

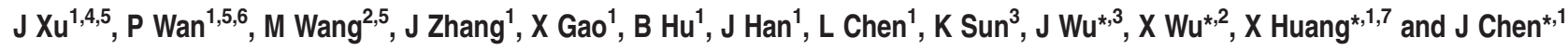

In mammals, spermatogonial stem cells (SSCs) arise from early germ cells called gonocytes, which are derived from primordial germ cells during embryogenesis and remain quiescent until birth. After birth, these germ cells migrate from the center of testicular cord, through Sertoli cells, and toward the basement membrane to form the SSC pool and establish the SSC niche architecture. However, molecular mechanisms underlying germ cell migration and niche establishment are largely unknown. Here, we show that the actin disassembly factor actin interacting protein 1 (AIP1) is required in both germ cells and Sertoli cells to regulate this process. Germ cell-specific or Sertoli cell-specific deletion of Aip1 gene each led to significant defects in germ cell migration after postnatal day 4 or 5 , accompanied by elevated levels of actin filaments (F-actin) in the affected cells. Furthermore, our data demonstrated that interaction between germ cells and Sertoli cells, likely through E-cadherin-mediated cell adhesion, is critical for germ cells' migration toward the basement membrane. At last, Aip1 deletion in Sertoli cells decreased SSC self-renewal, increased spermatogonial differentiation, but did not affect the expression and secretion levels of growth factors, suggesting that the disruption of SSC function results from architectural changes in the postnatal niche.

Cell Death and Disease (2015) 6, e1818; doi:10.1038/cddis.2015.182; published online 16 July 2015

In mammals, spermatogenesis and male fertility depend on the self-renewing and differentiating functions of spermatogonial stem cells (SSCs), which are regulated by cues from the niche microenvironment. ${ }^{1}$ During embryogenesis, the precursors of SSCs can be traced to primordial germ cells (PGCs) in the proximal epiblast at embryonic day 6.25 (E6.25), which migrate to genital ridge and together with somatic cells there to form the embryonic gonad. ${ }^{2}$ The PGCs then differentiate to gonocytes (also called prespermatogonia), proliferate for a brief period of time, and then remain mitotically quiescent until birth. ${ }^{3-5}$ After birth, these neonatal germ cells (gonocytes) located at the center of testicular cord become proliferative and relocate themselves from the center toward the basement membrane of each testicular cord. ${ }^{4,6}$ During the migration or relocation process, germ cells associate with and move through the Sertoli cells, the sole somatic cell type within the testicular cord and the major component of the SSC niche. After reaching the basement membrane at the periphery, most of these germ cells adopt a distinct morphology and become the undifferentiated spermatogonial population, which includes SSCs and other non-stem cell progenitors, ${ }^{7-9}$ supposedly in response to cues from the supporting cells. It has been suggested that the postnatal germ cell migration is crucial for the formation of SSC pool and the establishment of the SSC niche architecture. However, the mechanisms underlying these two processes are not well understood.

In neonatal mice, germ cells specifically express the cell adhesion molecule E-cadherin on the cell surface, ${ }^{10,11}$ whereas other adhesion markers including $\mathrm{N}$-cadherin and $\beta 1$-integrin were found in both germ cells and Sertoli cells. ${ }^{12-14}$ However, whether these adhesion molecules have specific roles in germ cells' outward migration and subsequent differentiation were not yet known. In Drosophila testis, the germline stem cells (GSCs) were shown to attach to the somatic hub cells (a major niche component) via membrane bound E-cadherin in both cell groups, and disruption of E-cadherin-mediated cell adhesion between GSCs and hub cells severely affected self-renewal and maintenance of GSCs. ${ }^{15,16}$ Moreover, a recent study showed that the actin polymerization regulator profilin is required to localize and maintain E-cadherin to the GSC-hub cell interface and is thus essential for the maintenance of GSCs. This result

\footnotetext{
${ }^{1}$ State Key Laboratory of Pharmaceutical Biotechnology and MOE Key Laboratory of Model Animals for Disease Study, Model Animal Research Center, Nanjing University, Nanjing, China; ${ }^{2}$ State Key Laboratory of Reproductive Medicine, Nanjing Medical University, Nanjing, China and ${ }^{3}$ Bio-X Institute, Shanghai Jiaotong University, Shanghai, China

${ }^{*}$ Corresponding author: J Chen or X Huang or X Wu or J Wu, State Key Laboratory of Pharmaceutical Biotechnology and MOE Key Laboratory of Model Animals for Disease Study, Model Animal Research Center, Nanjing University, 12 Xue-fu Road, Nanjing 210061, China. Tel/Fax: +86 25 58641507; E-mail: chenjiong @ nju.edu.cn or huangxx@shanghaitech.edu.cn or xinwu@njmu.edu.cn or jiwu@sjtu.edu.cn

${ }^{4}$ Present address: Nanjing Maternity and Child Health Care Hospital, Nanjing Medical University, Nanjing, China.

${ }^{5}$ These authors contributed equally to this work.

${ }^{6}$ Present address: School of Life science, Jiangxi Science \& Technology Normal University, Nanchang, China.

${ }^{7}$ Present address: School of Life Science and Technology, ShanghaiTech University, Shanghai, China.

Abbreviations: SSCs, spermatogonial stem cells; AIP1, actin-interacting protein 1; F-actin, actin filaments; PGCs, primordial germ cells; GSCs, germline stem cells; ADFs, actin-depolymerizing factors; HE, hematoxylin and eosin; MACS, magnetic-activated cell sorting; BTB, blood-testis barrier

Received 23.10.14; revised 18.5.15; accepted 19.5.15; Edited by M Agostini
} 
is consistent with findings in other systems that dynamics of actin cytoskeleton directly regulate the assembly and maintenance of E-cadherin-based cell adhesion. ${ }^{17}$ Interestingly, we have previously shown that actin interacting protein 1 (AIP1), an actin disassembly factor, regulates E-cadherin distribution and dynamics during a cell rearrangement process of the Drosophila eye disc. ${ }^{18}$ AIP1 has been shown to act together with cofilin/actin-depolymerizing factors to promote actin dynamics in various cellular processes, and it is highly conserved in all eukaryotes examined so far. ${ }^{19-24}$ Here, we utilized germ cell- or Sertoli cell-specific deletion of Aip1 (also known as $W d r 1$ ) in the murine testis to study the process of germ cell migration and SSC niche establishment.

\section{Results}

Aip1 deletion in Sertoli cells or germ cells caused severe defects in spermatogenesis. First, we utilized the Aip1 (Wdr1) conditional knockout mouse model (Aip $1^{f / / f f}$ ), which contained loxP sites flanking the exon 4 of the gene, leading to the premature termination of Aip1 translation (details of the conditional knockout construct has been reported by Yuan et al. $^{25}$ ). To achieve deletion of Aip1 specifically in early developing testis, we crossed the Aip $1^{f / / f l}$ with Amh (antimüllerian hormone)-cre mice that express the cre recombinase in Sertoli cells starting from embryonic day 15 (E15). ${ }^{26,27}$ To obtain germ cell-specific Aip1 knockout, we crossed Aip $1^{\mathrm{fl} / f l}$ with Vasa-cre mice that express cre in the germline beginning from $\mathrm{E} 15 .{ }^{28}$ Western blot analysis (with a previously referenced anti-AIP1 antibody. ${ }^{25}$ ) of THY1+ germ cells extracted from testes of postnatal day 7 (P7) Aip $1^{\mathrm{fl} /-}$ : $\mathrm{Vasa}^{+/ c r e}$ (referred to as geAip $1^{-/-}$subsequently) mice showed an almost complete removal of the AIP1 protein, indicating an efficient deletion of Aip1 in the germ cells (Figure 1a). Western blot of Sertoli cells from testes of P7 Aip $1^{f / f l}: A m h^{+/ c r e}\left(\right.$ seAip $\left.1^{-/}\right)$mice exhibited $60 \%$ reduction of AIP1 protein level, indicating a partial loss-of-function of Aip1 in the Sertoli cells (Figure 1b). Finally, the marked increase of F-actin levels, as shown by phalloidin staining, in the P7 geAip $1^{-1-}$ germ cells (Figure 1c) and P7 seAip $1^{-/-}$Sertoli cells (Figure 1d), are highly consistent with our previous characterization of Aip1's loss-of-function actin phenotypes in Drosophila and mouse. ${ }^{18,25}$

Hematoxylin and eosin (HE) staining revealed that the earliest observable defects occurred at P7 when a significant portion of germ cells in both geAip $1^{-1-}$ and seAip $1^{-/-}$testes failed to relocate to the basement membrane (Figure 2a). Further $\mathrm{HE}$ and immunofluorescence analyses from $\mathrm{P} 12$ to P35 showed that Aip1 ablation in the germ cells and Sertoli cells both caused severe defects in later stages of spermatogenesis and Aip 1 was likely required for the later proliferation and meiosis of germ cells (Figure 2 and Supplementary Figure 1).

Aip1 is required in both Sertoli cells and germ cells for the outward migration of germ cells to basement membrane. We next focused our analysis on the outward migration of germ cells between P2 and P7 for both geAip $1^{-1-}$ and seAip $1^{-1-}$ testes, as defect in this process is the earliest
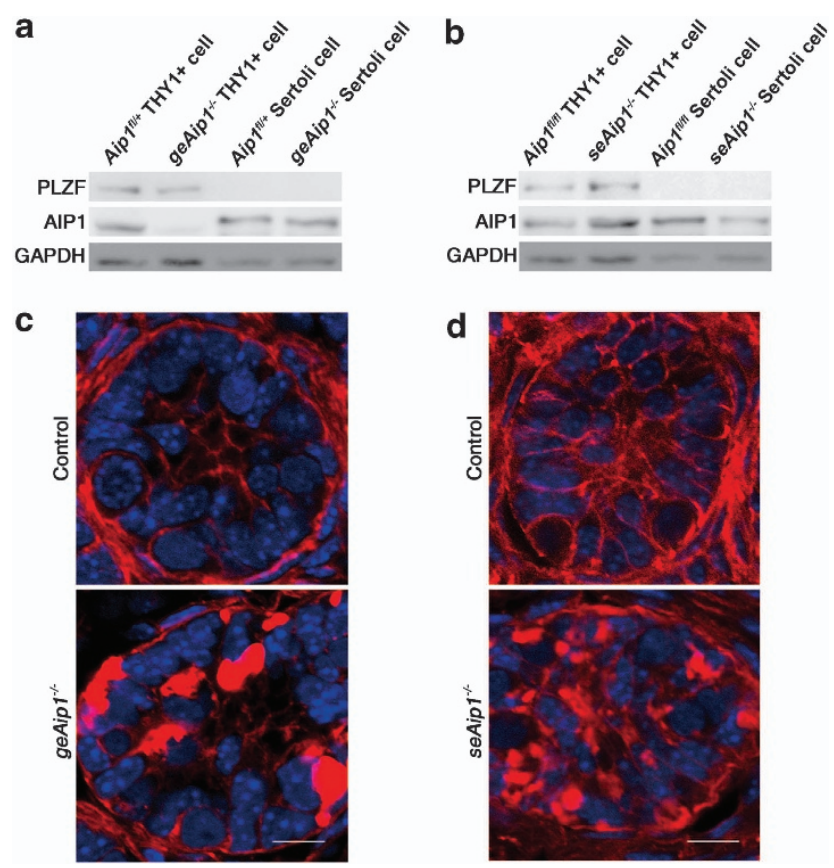

Figure 1 Reduction of AIP1 level and increase of F-actin level as the results of Aip1 deletion in either Sertoli cells or germ cells. ( $(\mathbf{a}$ and $\mathbf{b})$ Western blot analysis of AIP1 protein levels in isolated THY1+ germ cells from geAip $1^{-1-}$ testes at P7 (a) and isolated Sertoli cells from seAip1 $1^{-1-}$ testes at P7 (b). The control for (a) is THY1+ germ cells from Aip $1^{\mathrm{fl}+}$ testes and it is compared with THY1+ germ cells from geAip $1^{-/}$. The control for (b) is Sertoli cells from Aip $1^{\text {tl/ft }}$ testes and it is compared with Sertoli cells from seAip $1^{-1-}$ (c and d) Representative confocal images of phalloidin staining (red) of tissue sections from the P7 controls, geAip1 ${ }^{-1-}$ (c) and seAip1 $1^{-1-}$ (d) testes. Cell nuclei were stained with DAPI (blue). Scale bars: $10 \mu \mathrm{m}$

phenotype we detected and the subsequent severe spermatogenesis phenotypes could be the secondary consequence of earlier defects. To analyze the positioning of germ cells and Sertoli cells within the testicular cord, we immunostained sectioned testis tissues for PLZF (undifferentiated spermatogonia marker $\left.{ }^{29,30}\right)$, mouse vasa homolog $(\mathrm{MVH}$, general germ cell marker ${ }^{28}$ ) and GATA4 (Sertoli cell and Leydig cell marker ${ }^{31}$ ). In P2 control testis, most of round germ cells (gonocytes) labeled with MVH were shown to localize within the center of testicular cord, whereas Sertoli cells were positioned close to the basement membrane. At P4, a major portion of PLZF-labeled germ cells could be seen to relocate to the basement membrane. By P7, most of the germ cells had finished the outward migration, resulting in the large round spermatogonia intermingling with the smaller and irregularly shaped Sertoli cells adjacent to the basement membrane (Figure 3a). To quantify this process of germ cell migration, we counted both the number of germ cells that have reached the basal region of testicular cord $\left(N_{\text {basal }}\right)$ and the number of germ cells that have not reached the basement membrane and thus remain in the central region $\left(N_{\text {central }}\right)$ for each cross-sectional view of one testicular cord. In the control testes, we found that the total number of germ cells $\left(N_{\text {central }}+N_{\text {basal }}\right)$ per section increased from $2.21 \pm 0.19$ $(n=51)$ at P2 to $7.67 \pm 0.38$ at P7 $(n=52)$ (Figure $3 b)$. Such a 3.5-fold increase of germ cell number indicates that postnatal germ cells proliferate during their migration, which 
P14

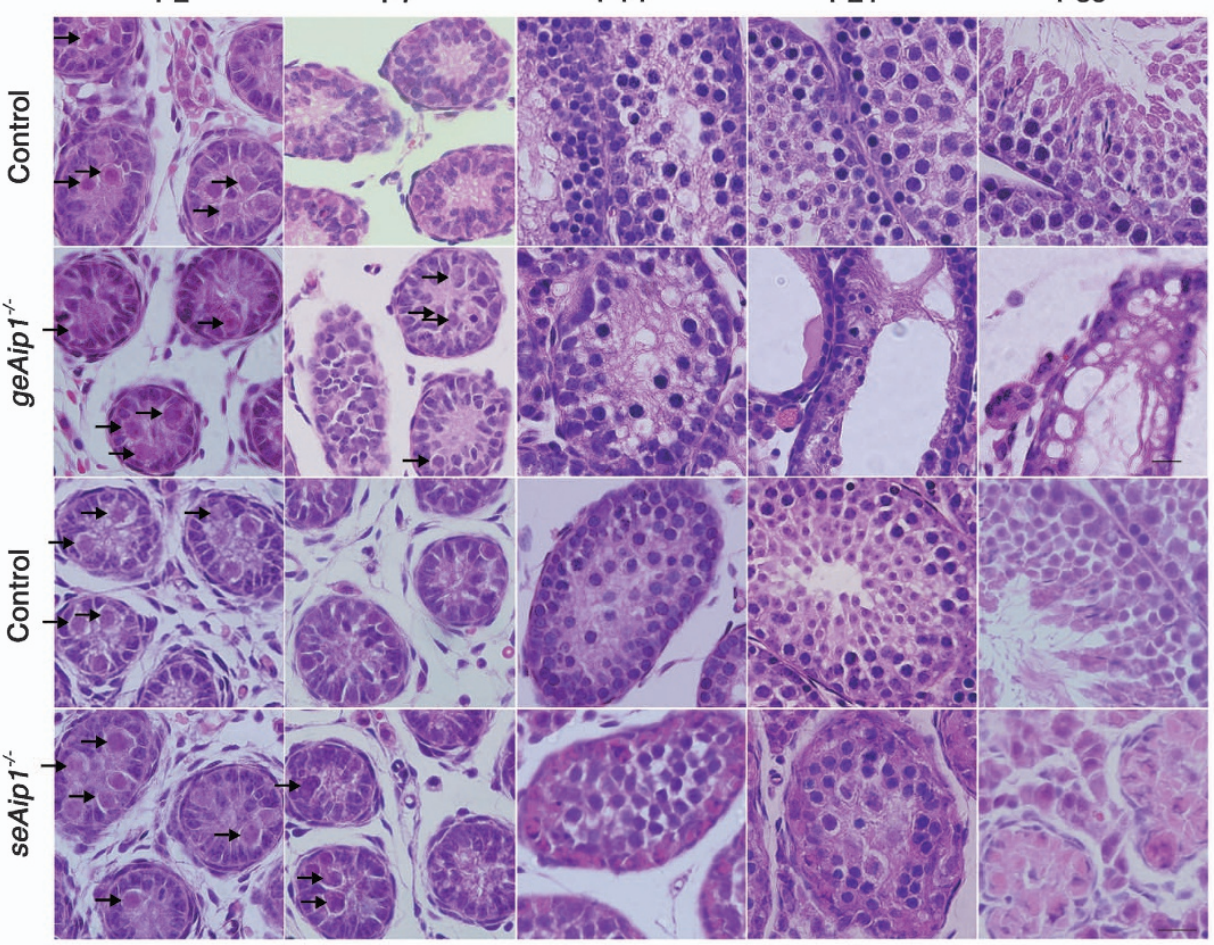

b

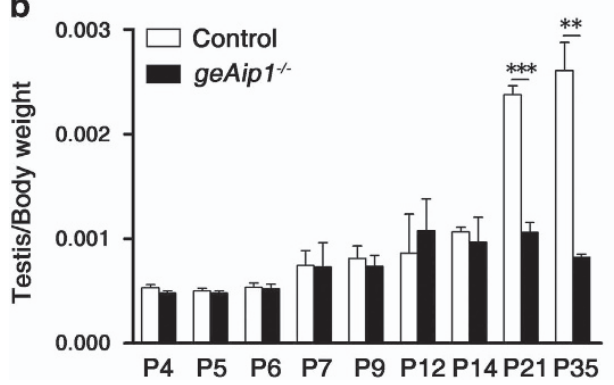

c

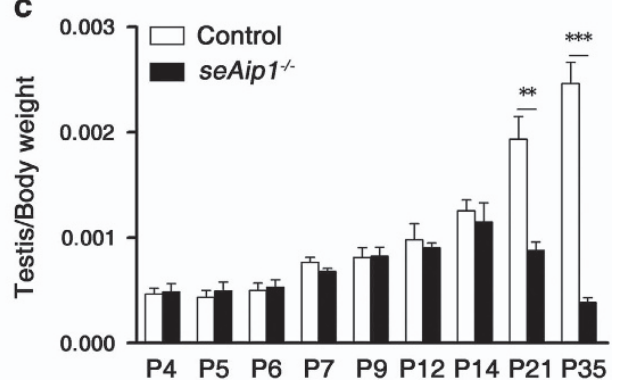

Figure 2 Severe spermatogenesis defects in geAip $1^{-1-}$ and seAip $1^{-1-}$ mice. (a) HE staining of tissue sections of testes from the controls, geAip $1^{-1-}$ and seAip $1^{-1-}$ mice at the indicated postnatal dates. No significant morphological defects can be seen in geAip $1^{-1-}$ and seAip $1^{-1-}$ testes at P2. At P7, germ cells in both geAip ${ }^{-1-}$ and seAip $1^{-1-}$ testes displayed abnormal positioning of a significant portion of germ cells in the central region of testicular cord, whereas almost all the germ cells in the control testes at P7 would have their migration and positioned themselves adjacent to the basement membrane. At P14, geAip $1^{-1-}$ and seAip $1^{-1-}$ testes displayed distinct phenotype. geAip ${ }^{-1-}$ testes at P14 displayed apparent reduction in the number of spermatocytes, whereas seAip $1^{-1}$ testes showed no significant reduction in the number of spermatocytes as compared with the control. At P21 and P35, in which round spermatids and spermatozoa begin to form, respectively, geAip $1^{-1-}$ testes exhibited marked reduction in the number of spermatids (at P21) and spermatozoa (at P35), indicating that spermatogenesis were effectively blocked at this stage. Similarly, seAip ${ }^{1-1-}$ testes also displayed severe reduction of spermatids and spermatozoa. And both geAip $1^{-1-}$ and seAip $1^{-1-}$ males are sterile. Arrows point to germ cells that were positioned in the central region of the testicular cord and had not reached the basement membrane. Scale bars: $10 \mu \mathrm{m}$. (b and $\mathbf{c}$ ) Ratios of testis/body weight were significantly reduced in the P21 and P35 geAip $1^{-1-}$ and seAip $1^{-1-}$ mice as compared with the controls. The strong size reduction in both geAip $1^{-1-}$ and seAip $1^{-1-}$ testes at P21 and P35 are consistent with the severe defects of spermatogenesis occurring in both knockout mice. Data are presented as means \pm S.E.M.; $n \geq 3$ in each group; ${ }^{* *} P<0.01,{ }^{* * \star} P<0.001$

is consistent with previous findings. ${ }^{8}$ In the mutant testes, the total number of germ cells increased in a similar fashion as the controls, from $2.28 \pm 0.21$ at $\mathrm{P} 2$ to $7.15 \pm 0.41$ at $\mathrm{P} 7$ (for geAip $1^{-/-}$) and from $3.29 \pm 0.27$ at P2 to $7.24 \pm 0.44$ at P7 (for seAip $1^{-/-}$), respectively (Figure $3 b$ and $\mathrm{c}$ ), suggesting that Aip1 deletion in either germ cells or Sertoli cells resulted in no major proliferation defects. Indeed, double staining with Ki67 (proliferative marker) and PLZF for the control and the mutant testes confirmed that no significant proliferation defect occurred in the germ cells in either geAip $1^{-/-}$or seAip $1^{-/-}$ testes (Supplementary Figures 2 and 3). However, the $N_{\text {basal }}$ value, which reflects the extent of germ cell migration, was decreased after P4 or P5 for geAip $1^{-1-}$ or seAip $1^{-1-}$ testes, respectively. From $\mathrm{P} 2$ to $\mathrm{P} 4$, there were no significant differences in the $N_{\text {basal }}$ or $N_{\text {central }}$ values between the mutant (geAip $1^{-/-}$or seAip $1^{-1-}$ ) and control testes, indicating that germ cells in both mutants migrate normally from P2 to P4. But beginning at $\mathrm{P} 5, \mathrm{~N}_{\text {basal }}$ of geAip $1^{-1-}$ testes was significantly decreased as compared with the control, and the decrease became most severe at P7. On the other hand, $N_{\text {central }}$ value began to display significant increase at P5 for geAip $1^{-1-}$ testes (Figure $3 b$ ). Similarly, reduction in $N_{\text {basal }}$ 

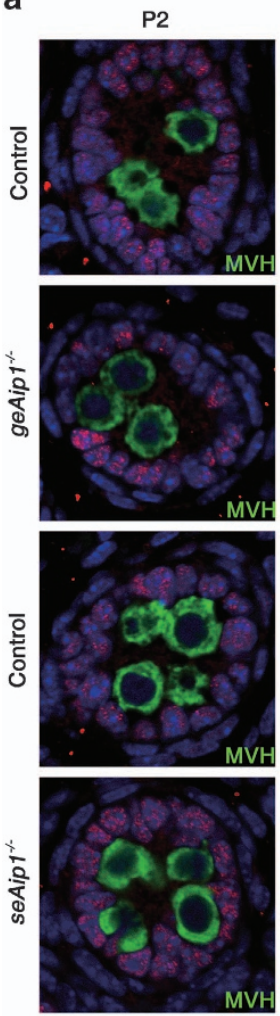

P4
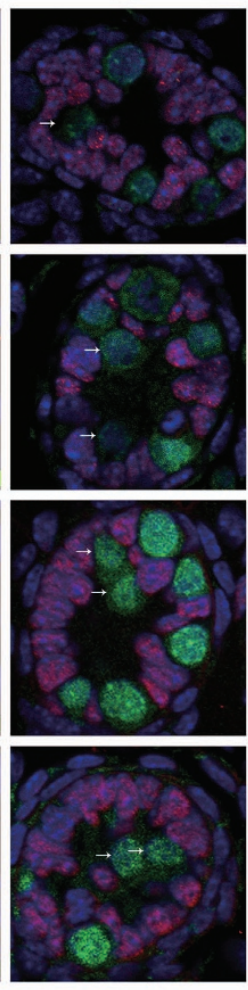

P5
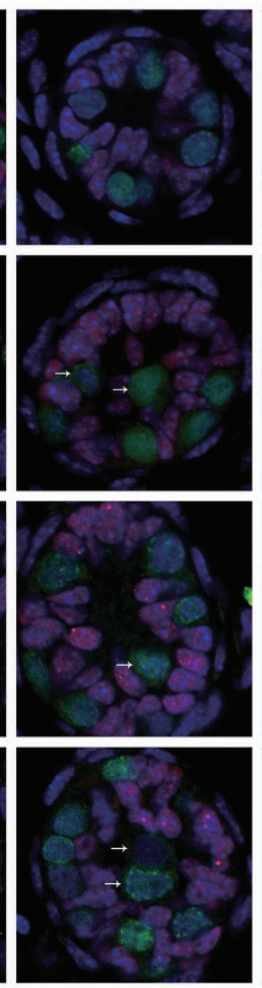

P6
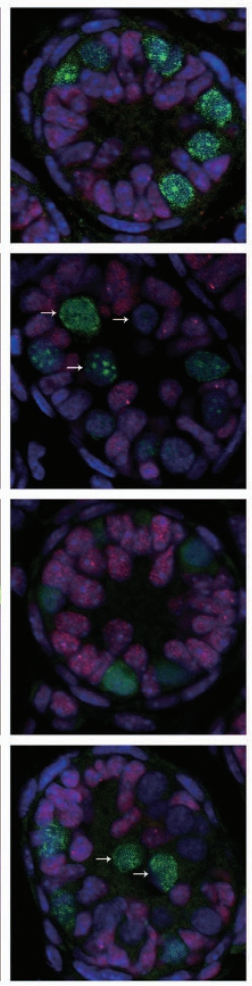

P7
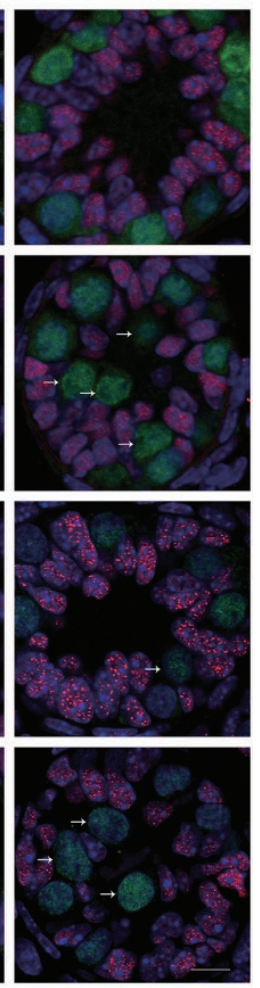

GATA4/PLZF/DAPI b

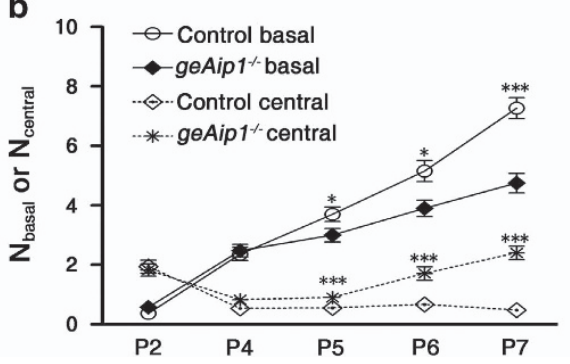

C

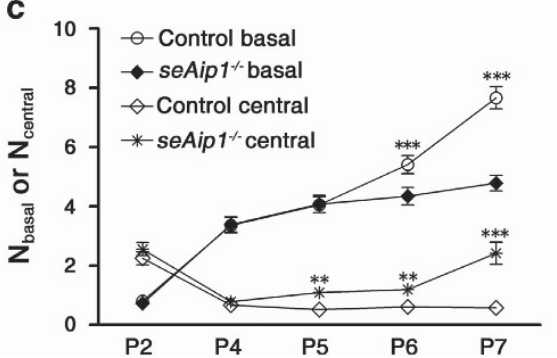

Figure 3 AIP1 is required in both germ cells and Sertoli cells for germ cells migration during postnatal testis development. (a) Germ cells from geAip1 ${ }^{-/-}$and seAip1 $1^{-/-}$ testes displayed significant defects in their migration as compared with their respective control. Control germ cells (labeled by MVH staining, green) at P2 mostly remained in the central of each tubule, whereas migratory germ cells (labeled by PLZF, green) were observed at P4-P5. By P6 or P7, most of the germ cells had reached and contacted the basement membrane of the testicular cord. In comparison, germ cells in the geAip $1^{-1-}$ and seAip $1^{-1-}$ testes showed similar localization at P2 and P4 as that of the controls, but displayed significant migration delay starting from P5 or P6. GATA4 staining labels the Sertoli cells (red). White arrows point to examples of germ cells that had not reached the basement membrane. Scale bar: $10 \mu \mathrm{m}$. (b and $\mathbf{c}$ ) Number of germ cells that were located at either the basal or central region of the testicular cord was quantified in geAip $1^{-1-}$ (b) and seAip $1^{-1-}$ (c) testes from P2 to P7 with respect to their controls. Each image was taken from a 5 - $\mu \mathrm{m}$-thick tissue section. A total of 51 tissue cross-sections were analyzed for each genotype at a certain time point (from three mice, except for P2 geAip $1^{-1-}$ ). Data are presented as means \pm S.E.M.; ${ }^{*} P<0.05,{ }^{\star \star} P<0.01,{ }^{* \star}{ }^{\star *} P<0.001$

value and increase in $N_{\text {central }}$ value also took place for seAip $1^{-1-}$ testes beginning at $\mathrm{P} 6$ and $\mathrm{P} 5$, respectively (Figure 3c). Taken together, these results demonstrate that Aip1 is autonomously required in the germ cells for their migration or relocation to the basement membrane after P4. Interestingly, Aip1 is also non-autonomously required in the supporting Sertoli cells for germ cell migration. Furthermore, germ cells' migration defects in both geAip $1^{-/-}$and seAip $1^{-/-}$ testes were not due to the effects of Aip1 ablation on cell proliferation of either germ cells or Sertoli cells.

We next determined what aspects of germ cell migration were prominently affected by Aip 1 deletion in either germ cells or Sertoli cells. Consistent with AIP1's roles in promoting actin dynamics in various model organisms, we found that Aip1 deletion caused actin disassembly defects and ectopic F-actin patches in Sertoli cells and germ cells within testes of seAip $1^{-/-}$and geAip $1^{-/-}$mice, respectively (Figure 1c and d). The ectopic F-actin phenotype in both $\operatorname{seAip1^{-/}}$ and geAip $1^{-/-}$testes began at P4 and became more severe in older mice (Supplementary Figure 4), which is consistent with the increasingly severe migration defects in the mutant testes. Immunostaining with antibody against $\beta$-actin also verified that the abnormal actin patches were enriched in the mutant testes (Figure $4 \mathrm{a}$ and b). In the P5 control testis, $\beta$-actin staining revealed that a significant portion of germ cells (15 out of 59 in P5 control) that have yet to reach the basement membrane 
a

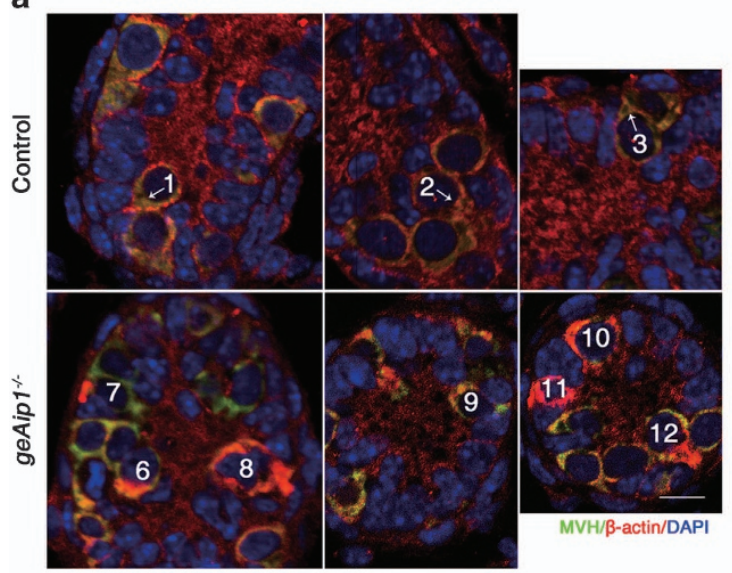

b

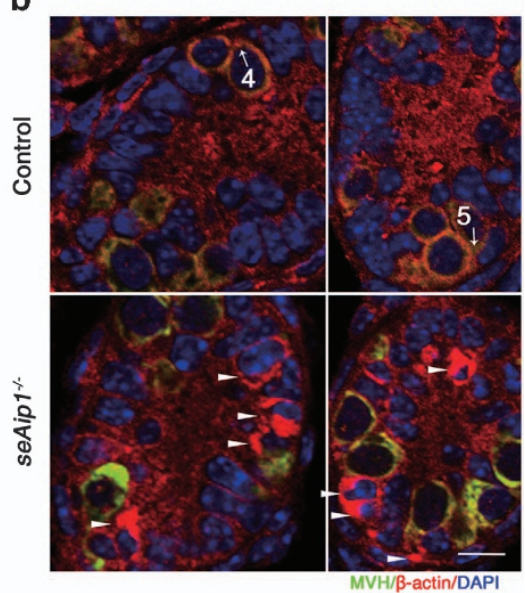

Figure 4 Aip1 deletion caused ectopic actin accumulations in the cell cortical regions and within lamellipodial protrusions during postnatal testis development. (a and b) Immunostaining of MVH (green) and $\beta$-actin (red) of testis tissue sections from P5 control, geAip $1^{-1-}$ and seAip $1^{-1-}$ mice. The cells numbered $1-5$ in the controls are wild-type germ cells migrating toward the basement membrane. Each arrow points to the migration direction and the polarized protrusion of the numbered germ cell. The cells numbered 6-12 are Aip1-deleted germ cells containing brightly stained actin patches in cell cortical regions. Arrowheads point to Aip1-ablated Sertoli cells with ectopic actin patches. Cell nuclei were stained with DAPI (blue). Scale bars: $10 \mu \mathrm{m}$

extended polarized and actin-rich lamellipodial protrusions toward the basement membrane (between Sertoli cells or between Sertoli cells and germ cells) during their migration (Figure $4 \mathrm{a}$ and B). In P5 geAip $1^{-1-}$ testes, these polarized lamellipodial protrusions with membrane-enriched actin staining were much less likely to be observed (15 out of 183). Instead, random (not polarized toward the basal region) protrusions and cell cortical regions filled with intensely stained actin patches were more frequently (62 out of 183) observed in germ cells that have not reached the basement membrane (Figure 4a). At last, in Sertoli cells of seAip1 $1^{-/-}$ testes, cell cortical regions were often observed to be filled with large ectopic patches of intense actin staining beginning at P4 (Figure 4b, Supplementary Figure 4). Together, these data indicate that actin disassembly mediated by AIP1 is required in the cell peripheral and lamellipodial regions of germ cells and Sertoli cells in the wild-type testis to promote outward migration of germ cells.

Aip1 deletion caused specific E-cadherin distribution defects in germ cells and Sertoli cells. As cell-cell and cell-substrate adhesions are critical for cell migration, we then examined the distribution patterns of cell adhesion markers in both control and mutant cells. We found that E-cadherin was strongly expressed in PLZF-labeled spermatogonia with a continuous and uniform E-cadherin staining on the cell membrane. In geAip $1^{-1-}$ testes, a significant portion ( $21 \%, n=15$ sections from three mice) of P7 germ cells displayed uneven and discontinuous membrane staining on the cell surface. Moreover, ectopic patches of strong E-cadherin staining were found near the cell surface (Figure 5a and c). Intriguingly, such defects of E-cadherin distribution in the germ cells were similarly found in seAip $1^{-1-}$ testes (Figure 5b), indicating that AIP1 loss-of-function in Sertoli cells had a non-autonomous effect on germ cells. In addition, we found ectopic E-cadherin staining on the membrane of a significant portion $(13.3 \%, n=23)$ of $\mathrm{P} 7$ mutant Sertoli cells (indicated by SOX9, a Sertoli cell marker ${ }^{32}$ ), whereas control Sertoli cells expressed only very little detectable E-cadherin staining (only 2\%, $n=18$ ) (Figure $5 \mathrm{~d}$ ). Culturing of control and seAip $1^{-/-}$Sertoli cells also confirmed the ectopic increase of E-cadherin level in the mutant Sertoli cells (Supplementary Figure 5). Surprisingly, significant upregulation of E-cadherin was also detected in Sertoli cells in the P7 geAip $1^{-1-}$ testes (Figure $5 \mathrm{c}$ and Supplementary Figure 6), indicating that Aip1-deleted germs cells had a non-autonomous effect on the wild-type Sertoli cells. Next, we found that $\mathrm{N}$-cadherin and $\beta 1$-integrin, two prominent adhesive markers that are uniformly localized on the cell surface of both germ cells and Sertoli cells, distributed normally for both types of cells in geAip $1^{-/-}$and seAip $1^{-1-}$ testes (Figure 6a-d). Taken together, these data suggested that the cytoskeletal changes due to AIP1 deficiency caused specific defects in E-cadherin localization for both germ cells and Sertoli cells and that E-cadherin or cytoskeletal defects in one cell type non-autonomously affected E-cadherin distribution of the other cell type.

Aip1 deletion in Sertoli cells affected SSC self-renewal and spermatogonial differentiation. The stem cell niche is essential for stem cell functions. To determine the effects of Sertoli cells on self-renewal of SSCs and differentiation of spermatogonia, we examined a variety of stem cell and cell differentiation markers in seAip $1^{-1-}$ testes at P4, P7, P9 and P12. We first identified SSCs among undifferentiated spermatogonia in both control and seAip $1^{-/-}$testes by double labeling of PLZF and OCT4 (a marker of SSC in testis $^{33,34}$ ). Consistent with previous reports, OCT4 was only expressed in a subset of PLZF-positive cells, ${ }^{35}$ and all OCT4positive cells are also PLZF-positive. Thus, we considered the OCT4 and PLZF double-positive cells as the SSCs. The number of SSCs in seAip $1^{-/-}$testes was not significantly different from control testes at P4 and P7. However, the number of OCT4 and PLZF double-positive cells as well as 
the PLZF-positive cells reduced significantly in P9 seAip $1^{-/-}$ testes when compared with the control (Figure 7a, b, d and e, Supplementary Figure 7). However, the number of all germ cells that were labeled by MVH (general germ cell marker ${ }^{36}$ ) remained unchanged in seAip $1^{-1-}$ testes at P7, P9 and P12 (Figure $7 \mathrm{~b}$ and $\mathrm{f}$ ), suggesting that the reduction of SSCs was
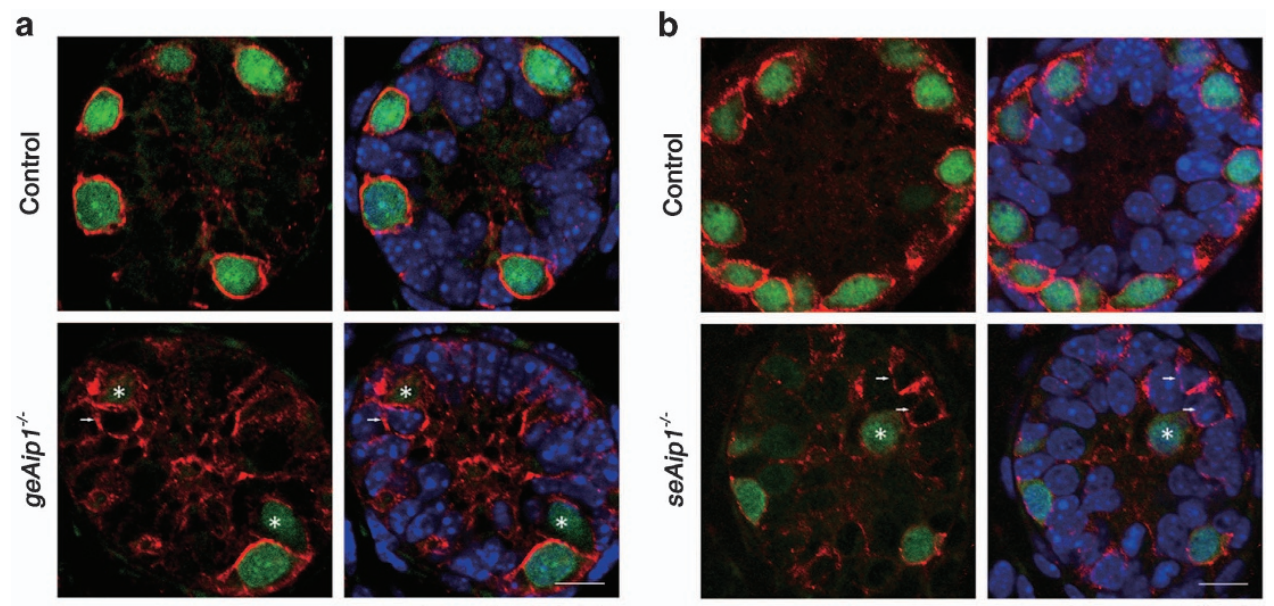

PLZF/E-cadherin/DAPI

PLZF/E-cadherin/DAPI
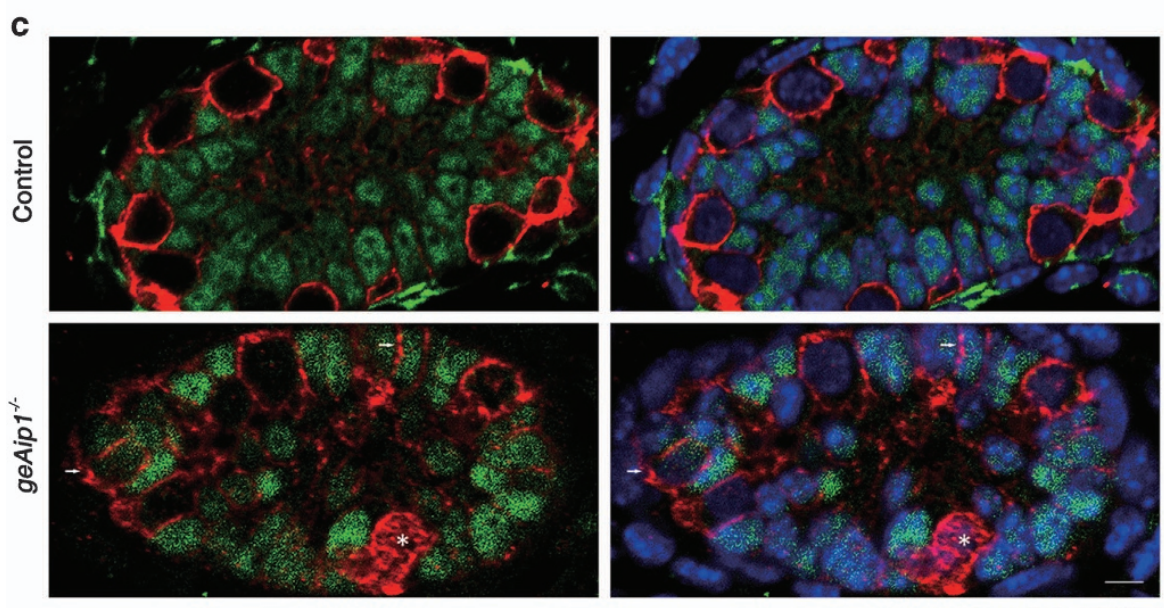

SOX9/E-cadherin/DAPI
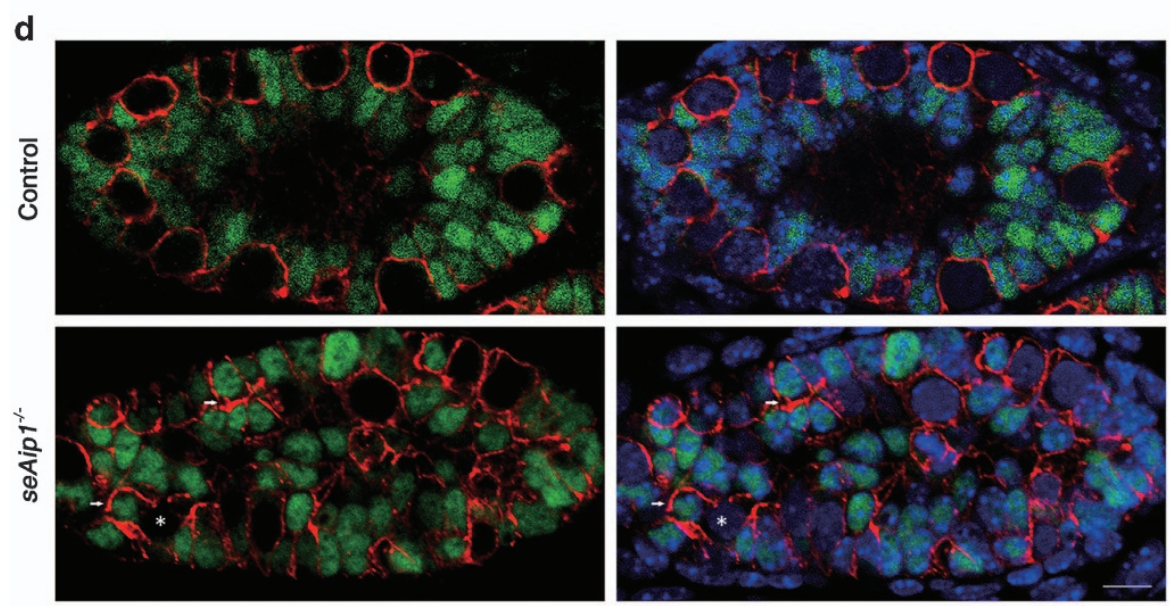

SOX9/E-cadherin/DAPI

Figure 5 Aip1 deletion in either germ cells or Sertoli cells caused alterations in E-cadherin distribution pattern in both germ cells and Sertoli cells. (a and $\mathbf{b}$ ) Co-immunostaining of E-cadherin (red) and PLZF (green) of testis sections from the P7 control, geAip1 ${ }^{-1-}$ (a) and seAip ${ }^{-1-}$ (b) mice. (c and d) Co-immunostaining of E-cadherin (red) and SOX9 (Sertoli cell marker, green) of testis sections from the P7 control, geAip1 ${ }^{-1-}$ (c) and seAip $1^{-1-}$ (d) mice. White arrows point to regions of Sertoli cells where E-cadherin level is upregulated, and the asterisk (*) signs mark germ cells whose E-cadherin distribution pattern is affected. Cell nuclei were stained with DAPI (blue). Scale bars: $10 \mu \mathrm{m}$ 

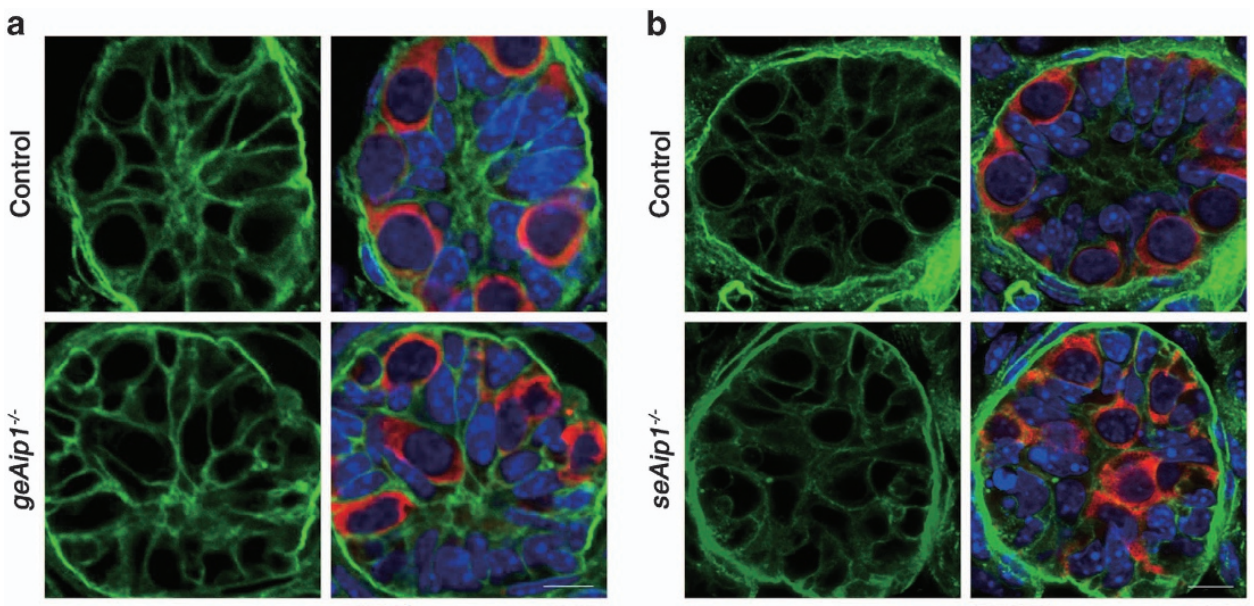

$\mathrm{MVH} / \beta 1$-integrin/DAPI

MVH/B1-integrin/DAPI
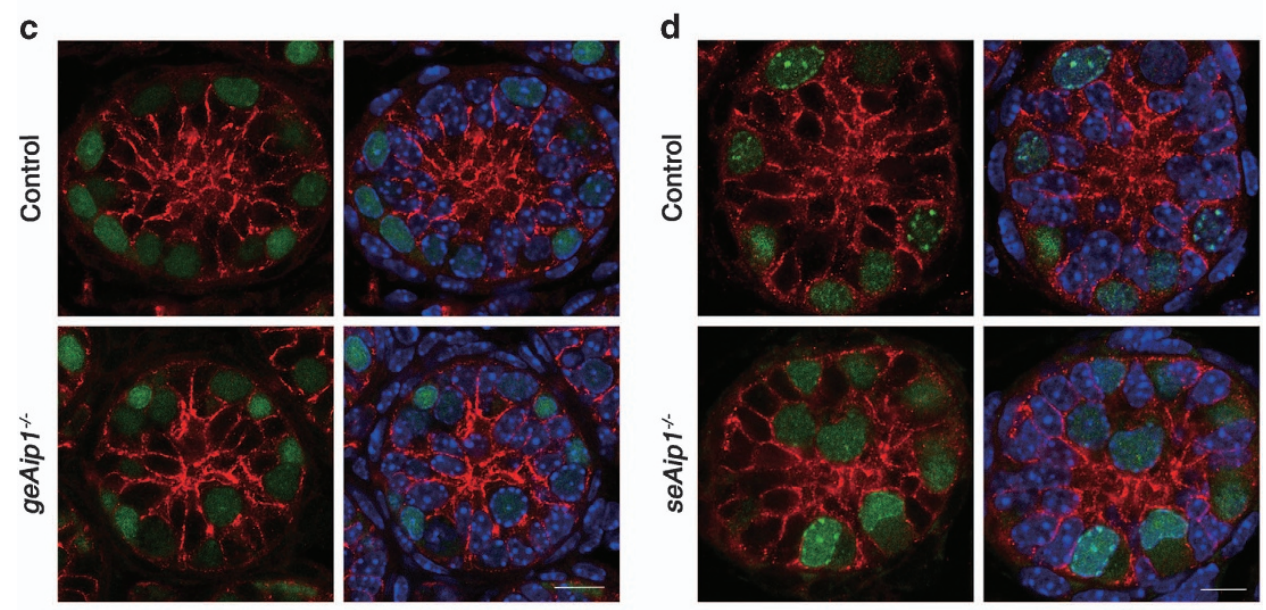

N-cadherin/PLZFDAPI
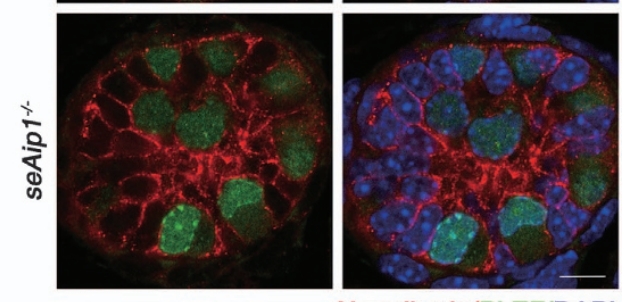

$\mathrm{N}$-cadherin/PLZF/DAPI

Figure 6 The distribution patterns of $\mathrm{N}$-cadherin and $\beta 1$-integrin were not significantly affected. (a and $\mathbf{b})$ Co-immunostaining of $\beta 1$-integrin (green) and MVH (red) of testis sections from the P7 control, geAip1 $1^{-1-}$ (a) and seAip1 ${ }^{-1-}$ (b) mice. (c and d) Co-immunostaining of N-cadherin (red) and PLZF (green) of testis sections from the P7 control, geAip1 $1^{-1-}$ (c) and seAip1 $1^{-1-}$ (d) mice. Scale bars: $10 \mu \mathrm{m}$

specific and was not a secondary consequence of reduction in the general germ cell population. At last, the marked reduction of SSC number at P9 cannot be explained by apoptosis, as there was no significant increase of activated Caspase 3 and PLZF double-positive cells in the P9 or P12 seAip $1^{-/-}$testes (Figure 7c and $\mathrm{g}$ ). Together, these data indicated that SSCs' self-renewing ability was nonautonomously affected by mutant Sertoli cells.

We next characterized the differentiation profile by real time (RT)-PCR analysis of the expression of $c-k i t,{ }^{37-39}$ the marker for differentiating spermatogonia, and Stra $8,{ }^{40}$ the marker for pre-meiotic germ cells in the whole testes. We found that the expression of both $c$-kit and Stra8 increased significantly in seAip $1^{-1-}$ testes at P9 (Figure 7h and i). Furthermore, flow cytometry data showed that the percentages of c-Kit+ and PLZF+ cells among the whole testes were significantly increased and decreased, respectively (Figure 7j and $k$, Supplementary Figure 7), confirming an increase of spermatogonial differentiation and decrease of spermatogonial self-renewal ability in the mutant testes. It was commonly understood that growth factors and chemokines secreted by Sertoli cells and other types of cells in the niche directly regulate the self-renewal and differentiation of SSCs. We next examined the expression levels of a variety of growth factors and chemokines that had been previously shown to affect SSC function. RT-PCR analysis showed that expression levels of $\mathrm{Gdnf},{ }^{41} \mathrm{CxCl} 12,{ }^{42-44} \mathrm{Fgf2},{ }^{45,46} \mathrm{Egf}^{47}$ and $\mathrm{Scf} \mathrm{f}^{4,49}$ displayed no significant difference between seAip $1^{-1-}$ and control testes (Supplementary Figure 8A). Furthermore, ELISA assay detected no significant alteration in the protein levels of secreted GDNF and FGF2 by isolated Sertoli cells from the P7 seAip $1^{-1-}$ testes (Supplementary Figure $8 \mathrm{~B}$ ). At last, transcript levels of Fshr, Lhcgr, Esr1 and Ar, which encode receptors for hormones $\mathrm{FSH}, \mathrm{LH}$, estrogen and androgen, respectively, ${ }^{50,51}$ were not significantly changed in seAip $1^{-1-}$ testes except for only mild reduction in levels of Fshr at P8 and P9, $A r$ at P9 (Supplementary Figure $8 \mathrm{C}$ ). These data suggested that the loss-of-function of AIP1 and the resulting SSC niche architecture change did not significantly alter the expression or secretion of growth factors or hormonal 
a
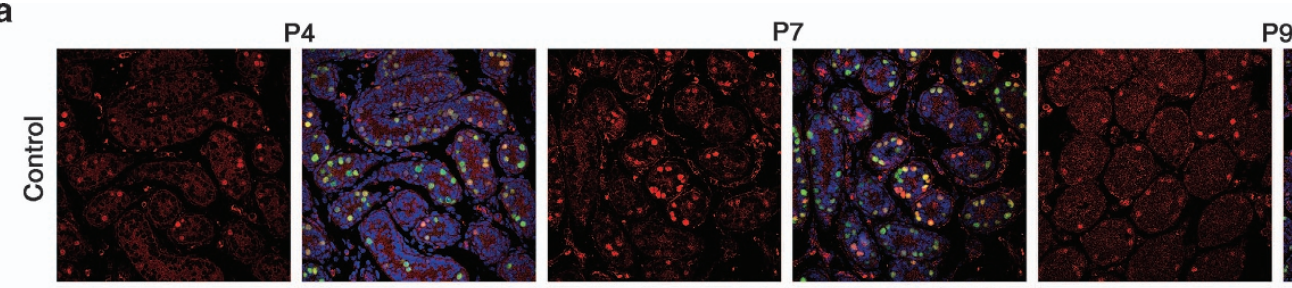

P9
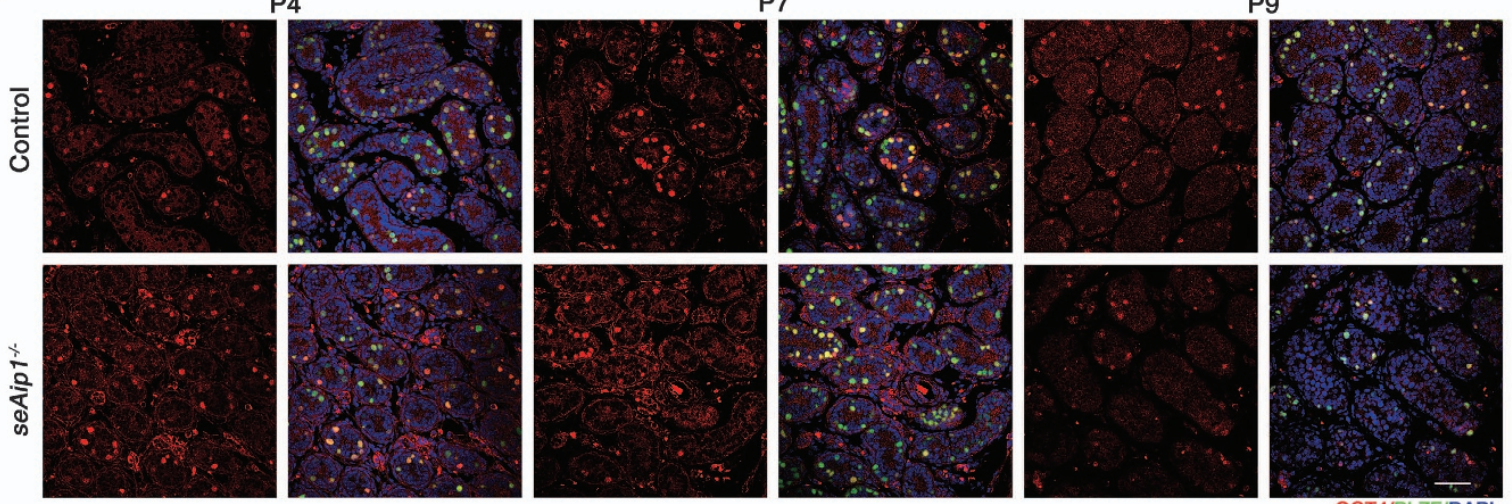

b

P7
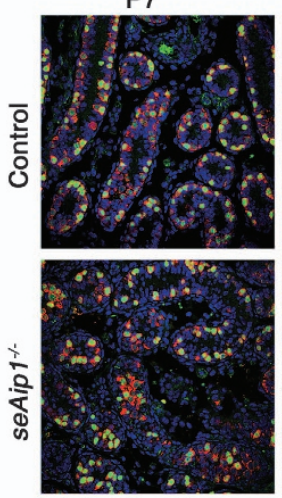

d

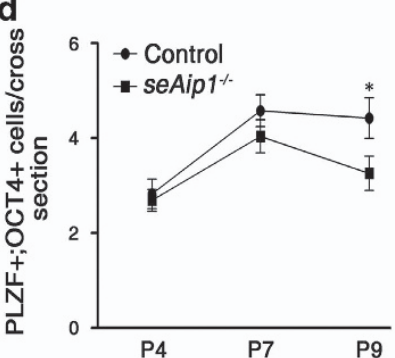

h

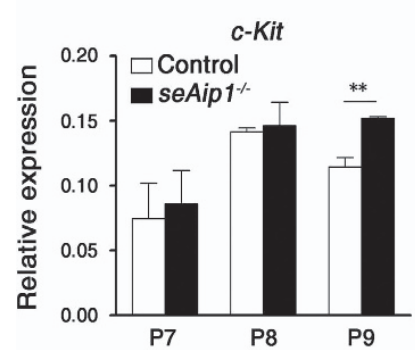

P9
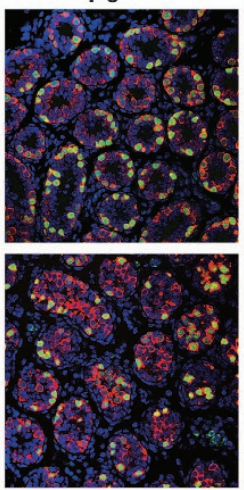

e

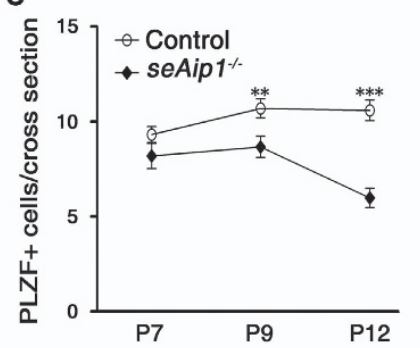

i
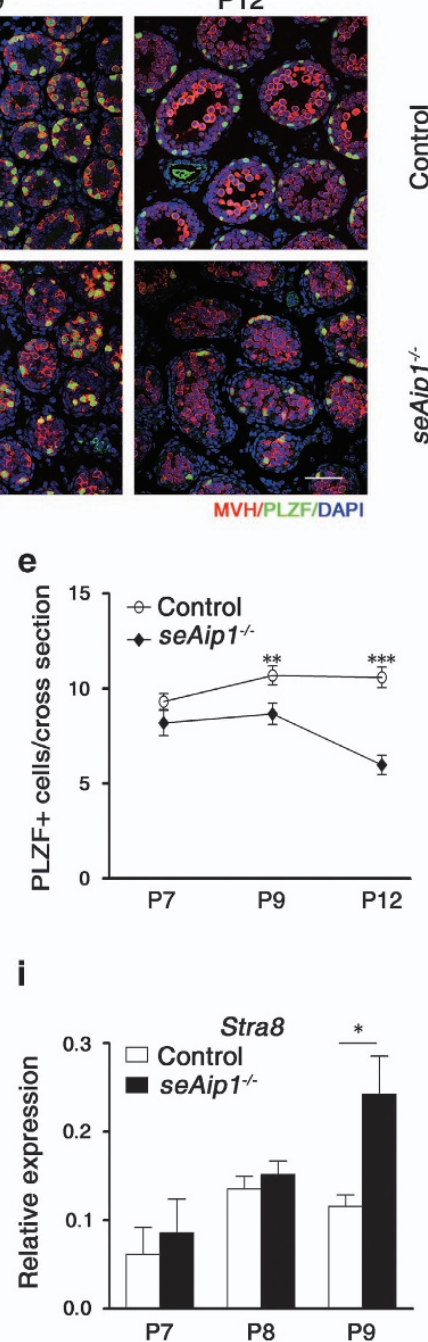

MVH/PLZF/DAPI
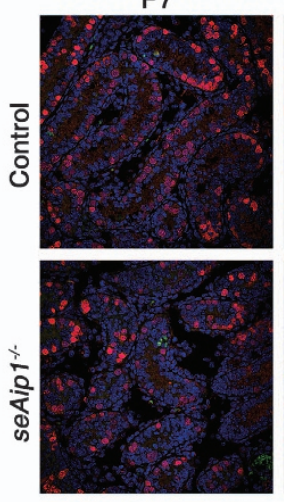

f

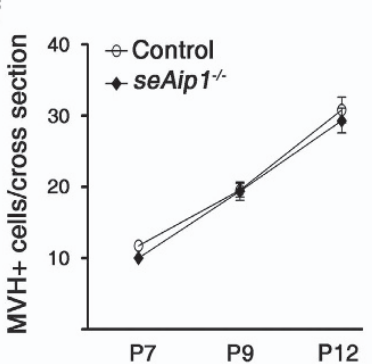

j

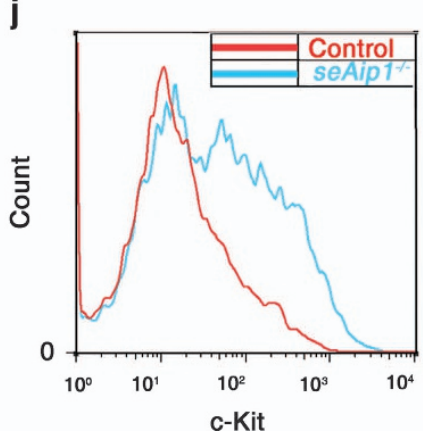

P9
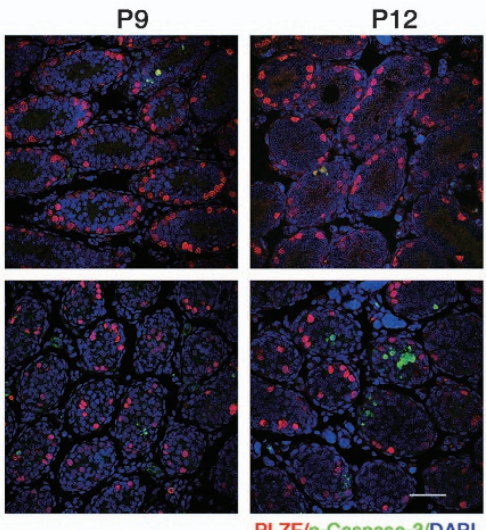

PLZF/p-Caspase-3/DAPI

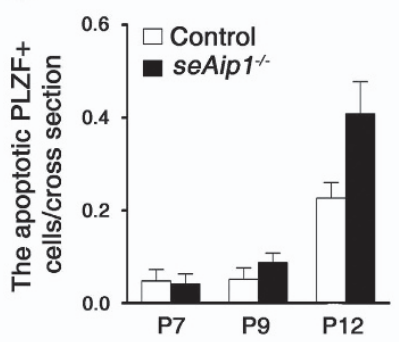

k

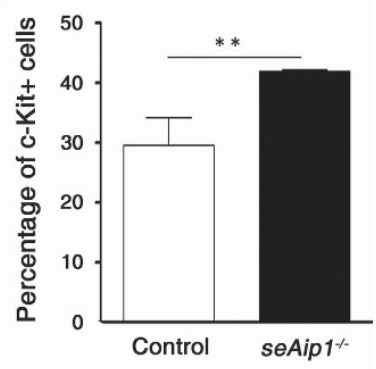

Figure 7 Aip1 deletion in Sertoli cells resulted in increase of spermatogonial differentiation and decrease of SSC self-renewal. (a) Co-immunostaining of OCT4 and PLZF in P4, P7 and P9 seAip $1^{-1-}$ and control testes. The result was quantified in (d). (b) Co-immunostaining of MVH and PLZF in P7, P9 and P12 seAip $1^{-1-}$ and control testes, with results quantified in (e) and (f). (c) Co-immunostaining of activated caspase-3 and PLZF in P7, P9 and P12 seAip1 ${ }^{-1-}$ and control testes, with results quantified in (g). (d-g) Quantification of SSC (d; PLZF and OCT double positive), undifferentiated spermatogonia (e; labeled by PLZF), germ cells (f; labeled by MVH) and apoptotic spermatogonia ( $\mathbf{g}$; PLZF and activated caspase-3 double positive) per cross-section of each testicular cord for both seAip $1^{-1-}$ and control mice at indicated postnatal dates. A total of 51 tissue cross-sections were analyzed for each genotype at a certain time point (from three mice, except for P12 in $\mathbf{c}$ and $\mathbf{g}$ ). (h and i) Relative expression levels of $c$-Kit (h) and Stra8 (i) from RT-PCR analysis. (j and k) Increased differentiation as demonstrated by flow cytometry analysis of the c-Kit+ cells. (j) Quantification of percentage of c-Kit+ cell from flow cytometry data. (k) Scale bars: $50 \mu \mathrm{m}$. Data are presented as means \pm S.E.M.; ${ }^{*} P<0.05,{ }^{\star \star} P<0.01,{ }^{* \star \star} P<0.001$ 
a
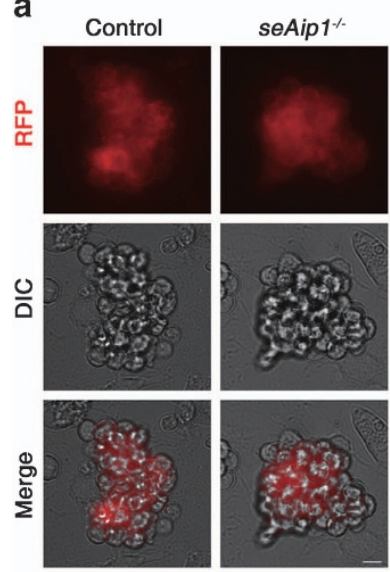

d

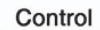
seAip $1^{-1-}$

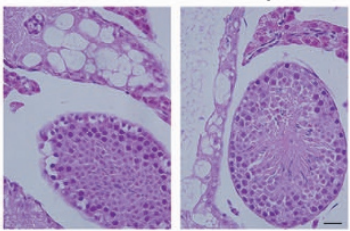

b

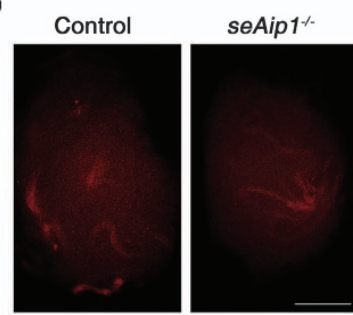

c

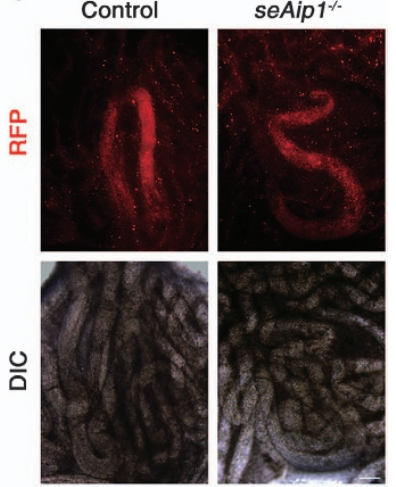

Figure 8 SSCs transplanted from defective niche maintained stem cell function and spermatogenesis potential both in vivo and in vitro. To trace the donor germ cells, especially in transplantation assay, seAip $1^{-1}$ - mice were crossed to Rosa26-mT/mG mice, a dual color reporter line, which possesses loxP sites on either side of a membrane-targeted tdTomato ( $m T$ ) cassette. After breeding with cre transgenic mice, the resulting offspring would have the $m T$ cassette deleted and the downstream membrane-targeted EGFP (mG) cassette expressed. (a) Representative THY1+ germ cell clumps from testes of seAip $1^{-1-}:$ Rosa26-mT/mG and Aip $1^{\text {t/flt }}:$ Rosa26-mT/ $m G$ mice that had been passaged for 16 times (2.5 months). (b, c) Representative images of recipient testes containing mT-labeled colonies (b) and mT-labeled seminiferous tubules from recipient testes (c). (d) Normal spermatogenesis shown by $\mathrm{HE}$ staining of the recipient testis that was transplanted with cells from seAip $1^{-1-}$ Rosa26-mT/mG and Aip $1^{t / / t / t}:$ Rosa26-mT/mG. Scale bars: $10 \mu \mathrm{m}$ in (a), $1 \mathrm{~mm}$ in (b), $0.2 \mathrm{~mm}$ in (c) and $20 \mu \mathrm{m}$ in (d)

receptors in Sertoli cells but rather caused changes in other aspects of the niche microenvironment, which probably includes alterations in the adhesive or architectural properties of the niche. Together, these results indicated that Aip1 deletion in Sertoli cells resulted in defects in postnatal SSC niche establishment.

Transplantation restored SSCs from seAip1 $1^{-1-}$ testes to their normal stem cell function. To determine whether the disrupted niche in seAip $1^{-1-}$ testes could result in irreversible effects on the SSCs, we performed germ cell transplantation, which was considered as effective means to examine the SSC function. ${ }^{13,52,53}$ THY1+ germ cells were isolated from P7-P8 testes of seAip1 $1^{-1-}:$ Rosa26-mT/mG and Aip $1^{f / f l}$ : Rosa26- $m T / m G$ donor mice by magnetic-activated cell sorting (MACS) to enrich $\mathrm{SSCs}^{54,55}$ (Supplementary Figures $9 \mathrm{~A}$ and B). The busulfan-treated C57BL/6 J×129sv F1 mice were used as germ cell transplant recipients. ${ }^{56}$ Two months after transplantation with sorted THY1+ cells, the germ cell colonies with red fluorescence (from $\mathrm{mT}^{57}$ ) derived from the donor testes were examined and counted (Figure $8 b$ and c). Surprisingly, germ cells from mutant mice produced 1.5-3.2 folds more colonies in the recipient testes than the control (Table 1). HE staining of the recipient testes showed that there were germ cells of different stages (Figure 8d), indicating that normal spermatogenesis of donor germ cells took place. Furthermore, in vitro proliferation assay showed that THY1+ germ cells from mutant testes retained the SSC potential to form colonies and had comparable growth rates as control germ cells (Figure 8a, Supplementary Figure 9). Taken together, these data demonstrated that defects in the niche microenvironment caused by Aip1 deletion in Sertoli cells did not produce irreversible damage to the SSC function.

\section{Discussion}

SSC's migration, in the context of homing to the germline niche, has recently been extensively studied. This migration event requires RAC1 and $\beta 1$-integrin, but not E-cadherin in the spermatogonia, and it also requires $\beta 1$-integrin in the Sertoli cells. ${ }^{13,53}$ However, during homing in the adult testis, SSCs need to cross the blood-testis barrier (BTB), which is composed of tight junctions between Sertoli cells. ${ }^{53}$ The migration of gonocytes occurs during the postnatal period and before the inter-Sertoli cells tight junction-BTB establishment (at $\mathrm{P} 12-\mathrm{P} 14^{53}$ ). At present, the underlying mechanism for postnatal germ cell migration is mostly unknown and it is supposed to be different from that of SSC homing. Here, we found that postnatal germ cell migration required AIP1 in both germ cells and Sertoli cells. Ectopic actin accumulations were apparent for both cell types beginning at P4. The onset of the actin defects correlated well with the onset of the migration defects. Our data indicated that impairment of actin disassembly could directly affect postnatal germ cell migration in two major ways.

First, ectopic actin patches were often found in the lamellipodia-like protrusions of migrating Aip $1^{-/}$germ cells, indicating the functional importance of the actin-based protrusions. A previous study reported that neonatal rat gonocytes with pseudopod extension were identified in the rat testis cell suspensions in vitro and these cells could colonize niche of recipient testis much more efficiently than gonocytes without pseudopod. ${ }^{58}$ But similar pseudopod extensions in gonocytes had not been detected in mouse. ${ }^{9}$ Our study provided important in vivo evidence that actin-rich protrusions existed in the postnatal germ cells and that these protrusions probably required robust actin turnover to drive germ cells' migration toward the basement membrane.

Second, the lack of actin dynamics could disrupt E-cadherin-mediated cell adhesion, leading to block of germ cells migration. Our data showed that the distribution pattern of E-cadherin but not of $\mathrm{N}$-cadherin and $\beta 1$-integrin was significantly affected in Aip $1^{-1-}$ germ cells and Sertoli cells. It is known that the membrane distribution and clustering of E-cadherin molecules are regulated by the dynamics of the underlying actin network. We have previously shown that the lack of AIP1 function in Drosophila caused disruption of E-cadherin remodeling and distribution in the adherens junction, often resulting in discontinuous membrane staining between two mutant cells. A recent study done in Drosophila testis revealed that disturbing actin polymerization by 
Table 1 Transplantation result

\begin{tabular}{|c|c|c|c|}
\hline & Control & $\operatorname{seAip1}^{-/-}$ & Fold change \\
\hline \multicolumn{4}{|l|}{ Experiment 1} \\
\hline Colony number $/ 10^{5} \mathrm{THY} 1+$ cells & 1.19 & 3.8 & 3.2 \\
\hline Colony length $(\mathrm{mm}) / 10^{5} \mathrm{THY} 1+$ cells & 1.30 & 5.24 & 4 \\
\hline \multicolumn{4}{|l|}{ Experiment 2} \\
\hline Colony number $/ 10^{5} \mathrm{THY} 1+$ cells & 5.96 & 9.23 & 1.55 \\
\hline Colony length $(\mathrm{mm}) / 10^{5} \mathrm{THY} 1+$ cells & 10.7 & 13.14 & 1.23 \\
\hline \multicolumn{4}{|l|}{ Experiment 3} \\
\hline Colony number $/ 10^{5} \mathrm{THY} 1+$ cells & 18.67 & 27.64 & 1.48 \\
\hline Colony length $(\mathrm{mm}) / 10^{5} \mathrm{THY} 1+$ cells & 31.3 & 57.25 & 1.83 \\
\hline
\end{tabular}

The total number of colonies from three to six different recipient testes was analyzed for each group

removing the function of profilin, an actin polymerizationpromoting factor, also caused disruption of E-cadherin staining in the interface between GSCs and the supporting hub cells. ${ }^{17}$ In agreement with the above reports, our data indicated that Aip1 deletion resulted in the disruption of E-cadherin distribution in the interface between germ cells and Sertoli cells, which could lead to their migration defects. Interestingly, Aip1 deletion in Sertoli cells also led to ectopic patches of E-cadherin staining near the cell membrane of Sertoli cells as well as discontinuous E-cadherin staining (but without ectopic patches) in the cell membrane of the spermatogonia. The autonomous effect of strong increase of membraneassociated or ectopically located E-cadherin could be explained if robust actin dynamics is required to promote fast turnover of membrane-associated E-cadherin in the wild-type Sertoli cells (probably via endocytosis). Indeed, actin dynamics has been shown to be critical for endocytosis. ${ }^{59}$ Furthermore, the non-autonomous effect on spermatogonia's E-cadherin distribution could be due to the altered pattern of E-cadherin localization in the adjacent Sertoli cells and the homophilic nature of the trans-membrane E-cadherin.

Our data from Aip 1 deletion in the Sertoli cells also provided important insights into how the SSC niche in the neonatal testis is established and how the niche influences SSC selfrenewal and differentiation. The niche is commonly thought of consisting of two parts, namely a growth factor milieu and architectural support. ${ }^{1}$ The Sertoli cell-specific ablation of Aip1 did not significantly alter the expression and secretion levels of growth factors in the Sertoli cells. However, germ cell migration toward the basement membrane was impaired, beginning at P5. This result suggested that disruption of germ cells migration results in defects in the establishment of the initial (neonatal) SSC niche, eventually leading to impairment of SSC function. This is possibly caused by the changes in niche architecture, which were manifested as alterations in the actin distribution in Sertoli cells and E-cadherin distribution in both the supporting Sertoli cells and spermatogonia. Interestingly, the architectural and adhesive alterations in the niche did not result in a permanent impairment of SSC function, as transplanting the SSCs taken from seAip $1^{-1-}$ testes can efficiently re-colonize recipient testes. This result suggested that disruption of SSC function as a result of architectural changes in the postnatal niche is reversible and that the
seAip $1^{-/-}$mice could serve as a good model to study the underlying mechanisms.

\section{Materials and Methods}

Mice. Mice were housed in standard cages in an Assessment And Accreditation of Laboratory Animal Care accredited SPF animal facility on a $12 \mathrm{~h} \mathrm{light/dark} \mathrm{cycle.}$ All animal protocols are approved by the Animal Care and Use Committee of the Model Animal Research Center of Nanjing University, the host for the National Resource Center for Mutant Mice in China. All mice used in this study were of a mixed 129/B6 background.

Histology and immunofluorescence. Testes were fixed with $4 \%$ paraformaldehyde, embedded in paraffin and transversely sectioned at a thickness of $5 \mu \mathrm{m}$. Tissue sections were stained with $\mathrm{HE}$ to examine histology. Immunofluorescence was carried out as previously described. ${ }^{60}$ Sections were counterstained with DAPI (Sigma-Aldrich, Saint-Quentin Fallavier, France), mounted in vectashield aqueous medium (Vector Laboratories, Inc., Burlingame, CA, USA), and analyzed using Olympus FV1000 confocal microscope. Primary antibodies used are: rabbit-anti-SOX9 (The original concentration is $200 \mu \mathrm{g} / \mathrm{ml}, 1: 50$ ), mouseanti-PLZF $(200 \mu \mathrm{g} / \mathrm{ml}, 1: 200)$, mouse-anti- $\beta$-actin $(100 \mu \mathrm{g} / \mathrm{ml}, 1: 200)$, mouse-antiGATA4 (200 $\mu \mathrm{g} / \mathrm{ml}, 1: 200)$, rabbit-anti-PLZF (200 $\mu \mathrm{g} / \mathrm{ml}, 1: 200)$, mouse-anti-OCT4 $(200 \mu \mathrm{g} / \mathrm{ml}, 1: 100)$ (Santa Cruz Biotechnology, Inc., Dallas, TX, USA, sc-20095, sc-28319, sc-81178, sc-25310, sc-22839, sc-5279), rabbit-anti-MVH $(0.8 \mathrm{mg} / \mathrm{ml}$, $1: 300)$, rabbit-anti-STRA8 $(0.8 \mathrm{mg} / \mathrm{ml}, 1: 300)$ (Abcam, Cambridge, UK; Ab13840, Ab49602), Rat-anti-Ki67 $(0.74 \mathrm{mg} / \mathrm{ml}, \quad 1: 200)$ (Dakocytomation, Glostrup, Denmark; M7249), rabbit-anti-Cleaved caspase-3 $(79 \mu \mathrm{g} / \mathrm{ml}, 1: 200)$ (Cell Signaling Technology Inc., \#9661), mouse-anti-E-cadherin ( $250 \mu \mathrm{g} / \mathrm{ml}, 1: 200)$, mouse-anti-Ncadherin $(250 \mu \mathrm{g} / \mathrm{ml}, 1: 200)$ (BD Biosciences, San Jose, CA, USA; 610182, 610920), rat-anti- $\beta 1$-integrin $(100 \mu \mathrm{g} / \mathrm{ml}, 1: 100)$ (EMD Millipore, MAB1997; Billierca, MA, USA). Cy3- Cy5- and FITC-conjugated antibodies (Jackson ImmunoResearch Laboratories, West Grove, PA, USA; 115-165-146 (1 mg/ml, $1: 200), 111-165-003(0.75 \mathrm{mg} / \mathrm{ml}, 1: 200), 715-175-150(0.25 \mathrm{mg} / \mathrm{ml}, 1: 200)$, $114-095-146(0.75 \mathrm{mg} / \mathrm{ml}, 1: 200), 111-095-144(0.75 \mathrm{mg} / \mathrm{ml}, \quad 1: 200))$ and AlexaFluro 488-conjugated antibodies (Invitrogen, Cergy Pontoise, France, A21208 $(2 \mathrm{mg} / \mathrm{ml}, 1: 200))$ were used as secondary antibodies. F-actin was labeled by AlexaFluor555 phalloidin (Invitrogen; A34055 (200 unit/ml, $1: 200)$ ).

Isolation and culture of THY1+ germ cells. Mouse testes were collected and digested by two-step enzymatic digestion procedure using collagenase and trypsin as described. ${ }^{52,61}$ In brief, after removing tunica albuginea of P7-P8 mouse testes, testicular cords were incubated with type IV collagenase (1 mg/ml; Sigma) in $37^{\circ} \mathrm{C}$ for $10 \mathrm{~min}$, following trypsin $(0.25 \%)$ and DNase $(7 \mathrm{mg} / \mathrm{ml})$ incubation for $5 \mathrm{~min}$. MACS was used to isolate THY1+ germ cells (Miltenyi Biotec, Bergisch Gladbach, Germany; anti-CD90.2, 130-049-101) as previously described. ${ }^{62}$ Cells were plated into wells of 12-well plate on mitotically inactivated STO (SIM mouse embryo-derived thioguanine and ouabain resistant) cell feeders $\left(5 \times 10^{4}\right.$ cells $\left./ \mathrm{cm}^{2}\right)$ as described. ${ }^{61,62}$ Cells were cultured with previously described optimized serum-free medium. ${ }^{61}$ All cultures were maintained at $37^{\circ} \mathrm{C}$ in a humidified $5 \% \mathrm{CO}_{2}$ incubator. The medium was changed every 2-3 days. 
Flow cytometry. The experiment was carried out as previously described..$^{43}$ In brief, the single-cell suspension of the P9 testes cells derived from two-step enzymatic digestion were incubated with the Anti-Mouse CD117 (c-Kit) APC-eFluor 780 antibody (0.125 $\mu \mathrm{g} /$ test) (eBioscience, Inc., San Diego, CA, USA). For the PLZF FACS, the P9 testes cell suspensions were first fixed and washed using the transcription factor buffer set (BD Biosciences, 562574) before incubated with PLZF (D-9) $(0.2 \mu \mathrm{g} / \mathrm{test})$ (sc-28319), then the Cy5-conjugated Goat-anti mouse antibody (Jackson ImmunoResearch Laboratories, 715-175-150 (0.5 $\mu \mathrm{g} / \mathrm{ml}, 1: 200))$ were incubated after washing by wash/perm provided in the buffer set. The stained cells were analyzed by FACS Calibur (BD Biosciences).

In vitro proliferation assay. After initial seeding density at $2 \times 10^{5}$ cell/well in 12-well plates, cultured THY1+ cells with a typical clump-forming morphology after 6 days culture were gently blew off from STO feeder layer and collected into a 15-m conical tube. After rinse and centrifugation, cells were dissociated with TrypsinEDTA to achieve a single-cell suspension. Following three washes in HBSS buffer, cells were concentrated and counted with Hemocytometer under microscope.

Transplantation assay. Aip $1^{f / / f t}: A m h^{+/ c r e}:$ Rosa26- $m T / m G$ and Aip $1^{f / / f t}$ : Rosa26-mT/mG mice were used as the mutant and control donors. MACS THY1 + cells were isolated from 12 testes of six mice at the age of P7-P8. $2.5 \times 10^{4} \mathrm{THY}$ + SSCs were transplanted into one C57BL/6 J×129sv F1 recipient testis, which were treated with busulfan at 6-8 weeks of age and received donor cells 2 months after busulfan treatment. The colony number was analyzed under the NIKON ECLIPSE E800 fluorescence microscopy.

RNA extraction and RT-PCR. The geAip $1^{-1-}$, seAip $1^{-1-}$ and control mouse testis were stripped of the tunica albuginea, placed in liquid nitrogen. Total RNAs were extracted using Trizol (Invitrogen, Carlsbad, CA, USA) according to the manufacturer's protocol. Three microgram of RNA was used for First-strand cDNA synthesize using RevertAid First Strand cDNA Synthesis Kit (Fermentas, Burlington, ON, USA). To quantify mRNA expression using RT-PCR, comparisons were made by normalizing the expression to that of glyceraldehyde-3-phosphate dehydrogenase (Gapdh) using Power-SYBR Green PCR Master mix (Takara, Takara Island, Japan) and StepOnePlus Real-Time System (ABI system, Cape Coral, FL, USA). The PCR conditions were $95^{\circ} \mathrm{C}$ for $30 \mathrm{~s}$, followed by 40 cycles of $95^{\circ} \mathrm{C}$ for $5 \mathrm{~s}$, $60^{\circ} \mathrm{C}$ for $31 \mathrm{~s}$. PCR was carried out using the primers listed in the Supplementary Table 1.

Sertoli cell isolation and ELISA. The Sertoli cells were isolated according to the protocol as previously described. ${ }^{63,64}$ in brief, digested testes cells were cultured in DMEM medium (Sigma-Aldrich) that was supplemented with sodium bicarbonate $(1.2 \mathrm{mg} / \mathrm{ml})$ and $10 \%$ fetal calf serum (Life Technologies, Carlsbad, CA, USA). After $24 \mathrm{~h}$ incubation in a humidified atmosphere at $37^{\circ} \mathrm{C}$ with $5 \% \mathrm{CO}_{2}$, the cells were treated with a hypotonic solution $(0.3 \times \mathrm{HBSS}, \mathrm{pH} 7.4)$ for $3 \mathrm{~min}$ to remove the spermatogenic cells adhering to the Sertoli cells. Then serum-free DMEM was added. After $24 \mathrm{~h}$ culturing, the medium was collected and concentrated. Elisa was performed using the concentrated sample and FGF2 Elisa kit (15.6 pg/ml $1000 \mathrm{pg} / \mathrm{ml}$, E90551 Mu, USCN, Wuhan, China) and GDNF Elisa kit $(78 \mathrm{pg} / \mathrm{ml} \sim 5000 \mathrm{pg} / \mathrm{ml}$, E90043 Mu, USCN).

Testicular cell co-culture. The experiment was performed as previously described with small modifications. ${ }^{65,66}$ In brief, the testicular cells from P4

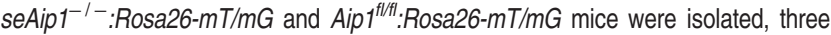
male pups for each group. And the testes were decapsulated in cold PBS, then collected and treated by the following sequential enzymatic digestion: $0.1 \%$ collagenase- $0.05 \%$ hyaluronidase $-0.1 \mathrm{mg} / \mathrm{ml}$ DNase for $20 \mathrm{~min}, 0.1 \%$ collagenase$0.1 \mathrm{mg} / \mathrm{ml}$ for another $20 \mathrm{~min}, 0.05 \%$ Trypsin-EDTA for $2 \mathrm{~min}$. After washing the cell suspension by trypsin inhibitor BSA $(0.65 \%$ and $0.05 \%$, respectively), the cells were plated at a density of $4.8 \times 10^{3}$ cells $/ \mathrm{mm}^{2}$ in the 24-well plate that had been precoated with gelatin and were cultured at $37^{\circ} \mathrm{C}$ in a $5 \% \mathrm{CO}^{2}$ atmosphere. The culture medium was DMEM supplemented with penicillin, streptomycin, $1 \mathrm{mM} \mathrm{Na}$ pyruvate, $1 \times$ nonessential amino acids, $3 \mathrm{mM} \mathrm{Na}$ lactate, $5 \mu \mathrm{g} / \mathrm{ml}$ transferrin and $50 \mathrm{ng} / \mathrm{ml}$ retinol. The medium was changed after $12 \mathrm{~h}$ plating and every 2 days thereafter.

Western blot. The THY1+ germ cells were harvested using the protocol described in the section of 'Isolation and Culture of THY1+ Germ Cells', and Sertoli cells were harvested using protocol described in 'Sertoli cell isolation and Elisa'.
Sertoli cells that were cultured in vitro for $24 \mathrm{~h}$ and isolated THY1+ cells were collected and lysed with lysis buffer (1\% NP-40, 0.5\% sodium deoxycholate, $0.1 \%$ SDS, $150 \mathrm{mM}$ sodium chloride, $0.1 \mathrm{mM}$ EDTA, $50 \mathrm{mM}$ Tris- $\mathrm{HCl}, \mathrm{pH} 7.2$, supplemented with $1 \mathrm{mM} \mathrm{NaF}, 1 \mathrm{mM}$ NaVO4, $1 \mathrm{mM}$ PMSF and Protease inhibitor cocktail (P3840, Sigma-Aldrich, St Louis, MO, USA)). Proteins concentration was measured using the BCA Protein Assay kit (23255, Pierce, Chicago, IL, USA). In total, $2-3 \mu \mathrm{g}$ of protein extracts were analyzed by immunoblotting with the following primary antibodies: rabbit-anti-AIP1 (22 $\mu \mathrm{g} / 150 \mu \mathrm{l}, 1: 500)$ (Protein tech group, Chicago, IL, USA; 13676-1-AP), mouse-anti-GAPDH (100 $\mu \mathrm{g} / \mathrm{ml}, \quad 1: 1000)$ (sc-32233), mouse-anti-PLZF (D-9) $(200 \mu \mathrm{g} / \mathrm{ml}, \quad 1: 500) \quad($ sc-28319). HRPconjugated secondary antibodies were used (Pierce, $31430(0.8 \mathrm{mg} / \mathrm{ml}, 1: 2000)$, $31460(0.8 \mathrm{mg} / \mathrm{ml}, 1: 2000))$.

\section{Conflict of Interest}

The authors declare no conflict of interest.

Acknowledgements. This work is supported by the grant from NSFC grants and 973 Program of China to X.H. $(31471400,2011$ CB944300) and NSFC grants to JC $(31071219,31271488)$.

1. Oatley JM, Brinster RL. The germline stem cell niche unit in mammalian testes. Physiol Rev 2012; 92: 577-595

2. Saitou M, Yamaji M. Primordial germ cells in mice. Cold Spring Harb Perspect Biol 2012; 4: $1-19$

3. Huckins $\mathrm{C}$, Clermont $\mathrm{Y}$. Evolution of gonocytes in the rat testis during late embryonic and early postnatal life. Arch Anat Histol Embryol 1968; 1: 341-354.

4. Clermont Y, Perey B. Quantitative study of the cell population of the seminiferous tubules in immature rats. Am J Anat 1957; 100: 241-267.

5. Culty M. Gonocytes, the forgotten cells of the germ cell lineage. Birth Defects Res C Embryo Today 2009; 87: 1-26.

6. Bellve AR, Cavicchia JC, Millette CF, Deborah AO, Bhatnagar YM, Martin D. Spermatogenic cells of the prepubertal mouse: isolation and morphological characterization. J Cell Biol 1977; 74: 68-85.

7. Kim S, Bardwell VJ, Zarkower D. Cell type-autonomous and non-autonomous requirements for Dmrt1 in postnatal testis differentiation. Dev Biol 2007; 307: 314-327.

8. Nagano R, Tabata S, Nakanishi Y, Ohsako S, Kurohmaru M, Hayashi Y. Reproliferation and relocation of mouse male germ cells (gonocytes) during prespermatogenesis. Anat Rec 2000; 258: 210-220

9. McLean DJ, Friel PJ, Johnston DS, Griswold MD. Characterization of spermatogonial stem cell maturation and differentiation in neonatal mice. Biol Reprod 2003; 69: 2085-2091.

10. Tokuda M, Kadokawa $\mathrm{Y}$, Kurahashi $\mathrm{H}$, Marunouchi $\mathrm{T}$. $\mathrm{CDH} 1$ is a specific marker for undifferentiated spermatogonia in mouse testes. Biol Reprod 2007; 76: 130-141.

11. Tolkunova EN, Malashicheva AB, Chikhirzhina EV, Kostyleva El, Zeng W, Luo J et al. E-cadherin as a novel surface marker of spermatogonial stem cells. Tsitologiia 2009; 51: 212-218.

12. Newton SC, Blaschuk OW, Millette CF. N-cadherin mediates Sertoli cell-spermatogenic cell adhesion. Dev Dyn 1993; 197: 1-13.

13. Kanatsu-Shinohara M, Takehashi M, Takashima S, Lee J, Morimoto H, Chuma S et al. Homing of mouse spermatogonial stem cells to germline niche depends on beta1-integrin. Cell Stem Cell 2008; 3: 533-542.

14. Palombi F, Salanova M, Tarone G, Farini D, Stefanini M. Distribution of beta 1 integrin subunit in rat seminiferous epithelium. Biol Reprod 1992; 47: 1173-1182.

15. Voog J, D'Alterio C, Jones DL. Multipotent somatic stem cells contribute to the stem cell niche in the Drosophila testis. Nature 2008; 454: 1132-1136.

16. Inaba M, Yuan H, Salzmann V, Fuller MT, Yamashita YM. E-cadherin is required for centrosome and spindle orientation in Drosophila male germline stem cells. PLoS One 2010; 5: e12473.

17. Shields AR, Spence AC, Yamashita YM, Davies EL, Fuller MT. The actin-binding protein profilin is required for germline stem cell maintenance and germ cell enclosure by somatic cyst cells. Development 2014; 141: 73-82.

18. Chu D, Pan H, Wan P, Wu J, Luo J, Zhu H et al. AlP1 acts with cofilin to control actin dynamics during epithelial morphogenesis. Development 2012; 139: 3561-3571.

19. Rodal AA, Tetreault JW, Lappalainen P, Drubin DG, Amberg DC. Aip1p interacts with cofilin to disassemble actin filaments. J Cell Biol 1999; 145: 1251-1264.

20. Clark MG, Amberg DC. Biochemical and genetic analyses provide insight into the structural and mechanistic properties of actin filament disassembly by the Aip1p cofilin complex in Saccharomyces cerevisiae. Genetics 2007; 176: 1527-1539.

21. Okada K, Blanchoin L, Abe H, Chen H, Pollard TD, Bamburg JR. Xenopus actin-interacting protein 1 (XAip1) enhances cofilin fragmentation of filaments by capping filament ends. J Biol Chem 2002; 277: 43011-43016. 
22. Ren N, Charlton J, Adler PN. The flare gene, which encodes the AIP1 protein of Drosophila, functions to regulate F-actin disassembly in pupal epidermal cells. Genetics 2007; 176 2223-2234.

23. Ono S. The Caenorhabditis elegans unc-78 gene encodes a homologue of actin-interacting protein 1 required for organized assembly of muscle actin filaments. J Cell Biol 2001; 152 1313-1320.

24. Kile BT, Panopoulos AD, Stirzaker RA, Hacking DF, Tahtamouni LH, Willson TA et al. Mutations in the cofilin partner Aip1/Wdr1 cause autoinflammatory disease and macrothrombocytopenia. Blood 2007; 110: 2371-2380.

25. Yuan B, Wan P, Chu D, Nie J, Cao Y, Luo W et al. A cardiomyocyte-specific Wdr1 knockout demonstrates essential functional roles for actin disassembly during myocardial growth and maintenance in mice. Am J Pathol 2014; 184: 1967-1980.

26. Lecureuil C, Fontaine I, Crepieux P, Guillou F. Sertoli and granulosa cell-specific Cre recombinase activity in transgenic mice. Genesis 2002; 33: 114-118.

27. Payne CJ, Gallagher SJ, Foreman O, Dannenberg JH, Depinho RA, Braun RE. Sin3a is required by sertoli cells to establish a niche for undifferentiated spermatogonia, germ cell tumors, and spermatid elongation. Stem Cells 2010; 28: 1424-1434.

28. Gallardo T, Shirley L, John GB, Castrillon DH. Generation of a germ cell-specific mouse transgenic Cre line, Vasa-Cre. Genesis 2007; 45: 413-417.

29. Buaas FW, Kirsh AL, Sharma M, McLean DJ, Morris JL, Griswold MD et al. Plzf is required in adult male germ cells for stem cell self-renewal. Nat Genet 2004; 36: 647-652.

30. Hobbs RM, Fagoonee S, Papa A, Webster K, Altruda F, Nishinakamura R et al. Functional antagonism between Sall4 and Plzf defines germline progenitors. Cell Stem Cell 2012; 10: 284-298.

31. Ketola I, Rahman N, Toppari J, Bielinska M, Porter-Tinge SB, Tapanainen JS et al. Expression and regulation of transcription factors GATA-4 and GATA-6 in developing mouse testis. Endocrinology 1999; 140: 1470-1479.

32. Matson CK, Murphy MW, Sarver AL, Griswold MD, Bardwell VJ, Zarkower D. DMRT1 prevents female reprogramming in the postnatal mammalian testis. Nature 2011; 476: 101-104.

33. Lei N, Hornbaker KI, Rice DA, Karpova T, Agbor VA, Heckert LL. Sex-specific differences in mouse DMRT1 expression are both cell type- and stage-dependent during gonad development. Biol Reprod 2007; 77: 466-475.

34. He Z, Jiang J, Hofmann MC, Dym M. Gfra1 silencing in mouse spermatogonial stem cells results in their differentiation via the inactivation of RET tyrosine kinase. Biol Reprod 2007; 77: 723-733.

35. Filipponi D, Hobbs RM, Ottolenghi S, Rossi P, Jannini EA, Pandolfi PP et al. Repression of kit expression by Plzf in germ cells. Mol Cell Biol 2007; 27: 6770-6781.

36. Tanaka SS, Toyooka Y, Akasu R, Katoh-Fukui Y, Nakahara Y, Suzuki R et al. The mouse homolog of Drosophila Vasa is required for the development of male germ cells. Genes Dev 2000; 14: 841-853

37. Prabhu SM, Meistrich ML, McLaughlin EA, Roman SD, Warne S, Mendis S et al. Expression of $c$-Kit receptor $\mathrm{mRNA}$ and protein in the developing, adult and irradiated rodent testis. Reproduction 2006; 131: 489-499.

38. Manova K, Nocka K, Besmer P, Bachvarova RF. Gonadal expression of c-kit encoded at the W locus of the mouse. Development 1990; 110: 1057-1069.

39. Yoshinaga K, Nishikawa S, Ogawa M, Hayashi S, Kunisada T, Fujimoto T et al. Role of c-kit in mouse spermatogenesis: identification of spermatogonia as a specific site of c-kit expression and function. Development 1991; 113: 689-699.

40. Zhou Q, Li Y, Nie R, Friel P, Mitchell D, Evanoff RM et al. Expression of stimulated by retinoic acid gene 8 (Stra8) and maturation of murine gonocytes and spermatogonia induced by retinoic acid in vitro. Biol Reprod 2008; 78: 537-545.

41. Meng X, Lindahl M, Hyvonen ME, Parvinen M, de Rooij DG, Hess MW et al. Regulation of cell fate decision of undifferentiated spermatogonia by GDNF. Science 2000; 287 1489-1493.

42. Yang Q, Kim D, Kaucher A, Oatley MJ, Oatley JM. CXCL12-CXCR4 signaling is required for the maintenance of mouse spermatogonial stem cells. J Cell Sci 2013; 126: 1009-1020.

43. Kanatsu-Shinohara M, Inoue K, Takashima S, Takehashi M, Ogonuki N, Morimoto H et al. Reconstitution of mouse spermatogonial stem cell niches in culture. Cell Stem Cell 2012; 11 : 567-578.

44. Yoon KA, Chae YM, Cho JY. FGF2 stimulates SDF-1 expression through the Erm transcription factor in Sertoli cells. J Cell Physiol 2009; 220: 245-256.

45. Zhang Y, Wang S, Wang X, Liao S, Wu Y, Han C. Endogenously produced FGF2 is essential for the survival and proliferation of cultured mouse spermatogonial stem cells. Cell Res 2012; 22: 773-776.

46. Ishii K, Kanatsu-Shinohara M, Toyokuni S, Shinohara T. FGF2 mediates mouse spermatogonial stem cell self-renewal via upregulation of Etv5 and Bcl6b through MAP2K1 activation. Development 2012; 139: 1734-1743.
47. Kurokawa S, Kojima Y, Mizuno K, Nakane A, Hayashi $Y$, Kohri K. Effect of epidermal growth factor on spematogenesis in the crytopchid rat. $J$ Urol 2005; 174: 2415-2419.

48. Ohta H, Yomogida K, Dohmae K, Nishimune Y. Regulation of proliferation and differentiation in spermatogonial stem cells: the role of c-kit and its ligand SCF. Development 2000; 127: 2125-2131.

49. Brannan Cl, Bedell MA, Resnick JL, Eppig JJ, Handel MA, Williams DE et al. Developmental abnormalities in Steel17H mice result from a splicing defect in the steel factor cytoplasmic tail. Genes Dev 1992; 6: 1832-1842.

50. Holdcraft RW, Braun RE. Hormonal regulation of spermatogenesis. Int J Androl 2004; 27: 335-342.

51. Petersen $\mathrm{C}$, Soder $\mathrm{O}$. The sertoli cell-a hormonal target and 'super' nurse for germ cells that determines testicular size. Horm Res 2006; 66: 153-161.

52. Ogawa T, Arechaga JM, Avarbock MR, Brinster RL. Transplantation of testis germinal cells into mouse seminiferous tubules. Int J Dev Biol 1997; 41: 111-122.

53. Takashima S, Kanatsu-Shinohara M, Tanaka T, Takehashi M, Morimoto H, Shinohara T. Rac mediates mouse spermatogonial stem cell homing to germline niches by regulating transmigration through the blood-testis barrier. Cell Stem Cell 2011; 9: 463-475.

54. Reding SC, Stepnoski AL, Cloninger EW, Oatley JM. THY1 is a conserved marker of undifferentiated spermatogonia in the pre-pubertal bull testis. Reproduction 2010; 139: 893-903.

55. Guo Y, Hai Y, Gong Y, Li Z, He Z. Characterization, isolation, and culture of mouse and human spermatogonial stem cells. J Cell Physiol 2014; 229: 407-413.

56. Bucci LR, Meistrich ML. Effects of busulfan on murine spermatogenesis: cytotoxicity, sterility, sperm abnormalities, and dominant lethal mutations. Mutat Res 1987; 176 259-268.

57. Muzumdar MD, Tasic B, Miyamichi K, Li L, Luo L. A global double-fluorescent Cre reporter mouse. Genesis 2007; 45: 593-605.

58. Orwig KE, Ryu BY, Avarbock MR, Brinster RL. Male germ-line stem cell potential is predicted by morphology of cells in neonatal rat testes. Proc Natl Acad Sci USA 2002; 99: 11706-11711.

59. Kaksonen M, Toret CP, Drubin DG. Harnessing actin dynamics for clathrin-mediated endocytosis. Nat Rev Mol Cell Biol 2006; 7: 404-414.

60. Xu J, Wang M, Gao X, Hu B, Du Y, Zhou J et al. Separase phosphosite mutation leads to genome instability and primordial germ cell depletion during oogenesis. PLOS One 2011; 6 : e18763.

61. Kubota $\mathrm{H}$, Brinster RL. Culture of rodent spermatogonial stem cells, male germline stem cells of the postnatal animal. Methods Cell Biol 2008; 86: 59-84

62. Kubota H, Avarbock MR, Brinster RL. Culture conditions and single growth factors affect fate determination of mouse spermatogonial stem cells. Biol Reprod 2004; 71: 722-731.

63. Wang $X$, Ying $P$, Diao F, Wang $Q$, Ye D, Jiang $C$ et al. Altered protein prenylation in Sertoli cells is associated with adult infertility resulting from childhood mumps infection. J Exp Med 2013; 210: 1559-1574.

64. Sun B, Qi N, Shang T, Wu H, Deng T, Han D. Sertoli cell-initiated testicular innate immune response through toll-like receptor-3 activation is negatively regulated by Tyro3, Axl, and mer receptors. Endocrinology 2010; 151: 2886-2897.

65. Orth JM, Boehm R. Functional coupling of neonatal rat sertoli cells and gonocytes in coculture. Endocrinology 1990; 127: 2812-2821.

66. Yu X, Sidhu JS, Hong S, Faustman EM. Essential role of extracellular matrix (ECM) overlay in establishing the functional integrity of primary neonatal rat Sertoli cell/gonocyte co-cultures: an improved in vitro model for assessment of male reproductive toxicity. Toxicol Sci 2005; 84 : 378-393.

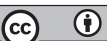

Cell Death and Disease is an open-access journal published by Nature Publishing Group. This work is licensed under a Creative Commons Attribution 4.0 International License. The images or other third party material in this article are included in the article's Creative Commons license, unless indicated otherwise in the credit line; if the material is not included under the Creative Commons license, users will need to obtain permission from the license holder to reproduce the material. To view a copy of this license, visit http://creativecommons.org/licenses/by/4.0/ 